\title{
Development of antibacterial compounds that block evolutionary pathways to resistance
}

\author{
Yanmin Zhang ${ }^{1,2 \#, ~ S o u r a v ~ C h o w d h u r y ~}{ }^{2 \#}$, João V. Rodrigues ${ }^{2}$, Eugene. Shakhnovich ${ }^{2}$ \\ ${ }^{1}$ School of Science, China Pharmaceutical University \\ 639 Longmian Avenue, Jiangning District \\ Nanjing, Jiangsu 211198 \\ P.R China \\ ${ }^{2}$ Department of Chemistry and Chemical Biology \\ Harvard University \\ 12 Oxford Street Cambridge MA 02138
}

\# contributed equally 


\begin{abstract}
Antibiotic resistance is a worldwide challenge. A potential approach to block resistance is to simultaneously inhibit WT and known escape variants of the target bacterial protein. Here we applied an integrated computational and experimental approach to discover compounds that inhibit both WT and trimethoprim (TMP) resistant mutants of E. coli dihydrofolate reductase (DHFR). We identified a novel compound (CD15-3) that inhibits WT DHFR and its TMP resistant variants L28R, P21L and A26T with $\mathrm{IC}_{50} 50-75 \mu \mathrm{M}$ against WT and TMP-resistant strains. Resistance to CD15-3 was dramatically delayed compared to TMP in in vitro evolution. Whole genome sequencing of CD15-3 resistant strains showed no mutations in the target folA locus. Rather, gene duplication of several efflux pumps gave rise to weak (about twofold increase in $\mathrm{IC}_{50}$ ) resistance against $\mathrm{CD} 15-3$. Altogether, our results demonstrate the promise of strategy to develop evolution drugs - compounds which block evolutionary escape routes in pathogens.
\end{abstract}




\section{Introduction}

Fast paced artificial selection in bacteria against common antibiotics has led to the emergence of highly resistant bacterial strains which potentially render a wide variety of antibiotics clinically ineffective. Emergence of these "superbugs" including ESKAPE (Enterococcus faecium, Staphylococcus aureus, Klebsiella pneumoniae, Acinetobacter baumannii, Pseudomonas aeruginosa, and Enterobacter spp.) (Peneş et al., 2017) call for novel approaches to design antibiotic compounds that act as "evolution drugs" by blocking evolutionary escape from antibiotic stressor. Currently drug development protocols and treatment strategies have limited success in addressing the issue of drug resistance as it overlooks the driving complex underlying evolutionary selection processes. The problem of drug resistance and its potential solutions constitutes important aspects of "Evolutionary medicine" (Stearns, 2012; Williams and Nesse, 1991). Apart from addressing specific questions involving disease mechanism and their ontogeny, evolutionary medicine takes a more comprehensive approach and aims to address evolutionary questions such as selective advantage of a phenotype and/or the associated phylogeny (Nesse and Stearns, 2008). This makes it immensely pertinent in the context of the problem of drug resistance which is primarily an evolutionary escape under conditions of selection pressure exerted by chemical stressors viz. antibiotics. Application of evolution principles has been promising in the studies of infectious diseases, aging and cancer therapy (Nesse and Stearns, 2008). Evolution of cancer cells and their complex ways to evade the anticancer therapeutics in many ways resembles the evolutionary selection of resistant bacterial cells evading the antibiotic action (Stearns, 2012). Development of "evolution drugs" - compounds that block or slow down escape routes to resistance - is inspired by recent progress in understanding of evolutionary dynamics of pathogen escape from stressors (Chakraborty and Barton, 2017; Gong et al., 2013; Klein et al., 2018; Marchi et al., 2019; Rotem et al., 2018) Recent report of compounds modulating proteins controlling mutation rates is an interesting lead in that direction (Ragheb et al., 2019). Using evolution drugs to slow down evolutionary dynamics of pathogen or cancer escape can help tilt the balance of evolutionary arms-race in favour of the host immune system.

Selectively targeting bacterial proteins which are critical to essential bacterial life processes like cell wall biosynthesis, translation, DNA replication etc. with novel compounds forms the basis of antibiotics development programs. Dihydrofolate reductase (DHFR) is one such protein, which, due to its critical role in nucleotide biosynthesis, has been a central antibacterial and anticancer drug target (Lin and Bertino, 1991; Schweitzer et al., 1990). Based on the chemical scaffold, DHFR inhibitors can be divided into classical and non-classical ones. The classical DHFR inhibitors generally contain a 2,4-diamino-1,3-diaza pharmacophore group (Bharath et al., 2017) which constitute structural analogues of its substrate dihydrofolate and competitively bind the receptor-DHFR active site. Inhibitors of this type such as methotrexate (MTX) (Bleyer, 2015) 
and pralatrexate (PDX) (Izbicka et al., 2009) are approved as anticancer drugs. In addition, predominant classes of inhibitors derived from dihydrofolate analogues also include diaminoquinazoline, diaminopyrimidine, diaminopteridine, and diaminotriazines (Bharath et al., 2017). Non-classical antifolate drugs like trimethoprim (TMP)(Finland and Kass, 1973) trimetotrexate (TMQ) (Lin and Bertino, 1991), that interact selectively with bacterial but not human DHFR are approved as antibacterial drugs. Without the solvent accessible group of glutamic acid, they are more fat-soluble, passively diffuse into cells, and are also not substrates for folylpolyglutamate synthetase enzymes. However, due to rapid emergence of resistant mutations in DHFR, the development of drug resistance to antifolate antibiotics belonging to any of the above-mentioned classes presents a significant challenge (Huovinen et al., 1995). Nevertheless, TMP and other non-classical anti-folates can be attractive templates for bringing in modifications in the structure of DHFR inhibitors and derive novel DHFR inhibitors (Wróbel et al., 2020).

In general, both clinical and in vitro studies have shown that accumulation of point mutations in critical amino acids residues of the binding cavity represent an important mode of trimethoprim resistance. Factors associated with TMP resistance are far more complex in the clinical isolates. A recent study has shown the contribution of mobile dfrA genes to TMP resistance in the emerging pathogen Acinetobacter baumannii and its association to chromosomal folA genes in rapidly mobilizing novel mutations (Sánchez-Osuna et al., 2020). The study shows how sulfonamide resistance in general extends to TMP with generalized chromosomal resistance determinants predating the origin of several genera and several clusters of resistance genes disseminated broadly among clinical isolates. Further, whole-genome sequencing of TMPresistant E.coli clinical isolates showed the contribution of dfrB genes towards clinical TMP resistance (Toulouse et al., 2017). Thus, mechanisms leading to TMP resistance could be extremely diverse and complex. These complications notwithstanding, in our current study we focus on a simplified experimental model system of in vitro evolution whereby resistant phenotypes emerge from point mutations in the folA locus as outlined in detail below.

Point mutations conferring resistance in bacteria to anti-DHFR compounds are primarily located in the folA locus that encodes DHFR in E. coli (Oz et al., 2014; Tamer et al., 2019; Toprak et al., 2012) making DHFR an appealing target to develop evolution antibiotic drugs. A possible approach is to design compounds that can inhibit the wild type (WT) DHFR along with its resistant variants thus blocking multiple evolutionary pathways towards drug-resistance. In this work, we developed an integrative computational modeling and biological evaluation workflow to discover novel DHFR inhibitors that are active against WT and resistant variants. Structurebased virtual screening (SBVS) including molecular docking with subsequent validation by molecular dynamics (MD) (Cheron and Shakhnovich, 2017; Leonardo et al., 2015; Liu et al., 2020; Zhang et al., 2018) were used to screen a large compound database. We found a series of DHFR inhibitors with novel scaffolds that are active against both the WT and several mutant DHFR proteins and are cytotoxic against WT E. coli along with E. coli strains with 
chromosomally incorporated TMP resistant DHFR variants (Palmer et al., 2015a). Those inhibitors are more potent against the escape variants than the WT DHFR. This makes them promising candidates for further development of next generation of antibiotics that prevent fast emergence of resistance.

\section{Results}

\section{In silico search for potent broad DHFR inhibitors}

The key objective of our approach is to find compounds that simultaneously inhibit WT and drug resistant variants of a target protein. Firstly, we developed an integrative computational workflow including molecular docking, molecular dynamics and evaluation of protein-ligand interaction along with Lipinski's rule of five (Manto et al., 2018) filter to screen two commercial databases that include about 1.8 million compounds (Figure 1A). First, we assessed which conformation of M20 loop of DHFR (closed, open or occluded) should be used for molecular docking. To that end we evaluated which conformation of the M20 loop in the target structure gives rise to best agreement between docking score and experimental binding affinity for known DHFR inhibitors. By classification (see Method and Materials: Crystal structure selection), we selected four representative crystal structures (closed: PDB 1RX3, open: PDB 1RA3, occluded: PDB 1RC4 and PDB 5CCC, Figure S1) as putative target structures for docking. Using the closed conformation of M20 loop (PDB 1RX3), we were able to recover the largest proportion of known inhibitors (Table 2 and Figure S2-S3). Therefore, the closed conformation of M20 loop (PDB 1RX3) was adopted as the most representative crystal structure for the initial SBVS of compound databases for novel broad range inhibitors. It was also used for subsequent in-depth evaluation of most promising candidates using molecular dynamics simulation. The detailed discussion of the rationale behind selection of closed conformation is provided in the Method and Materials: Crystal structure selection. 
A

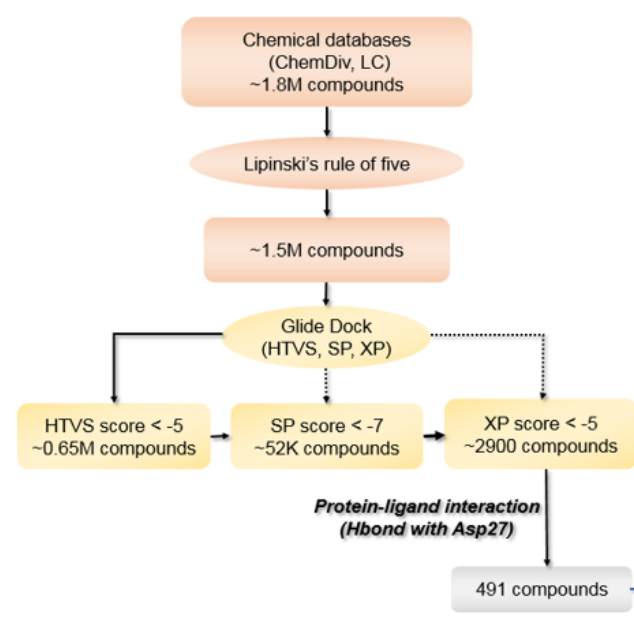

\section{B}

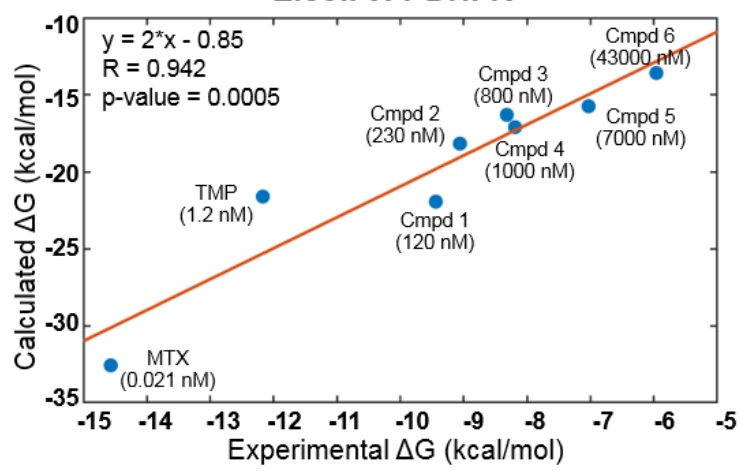

C
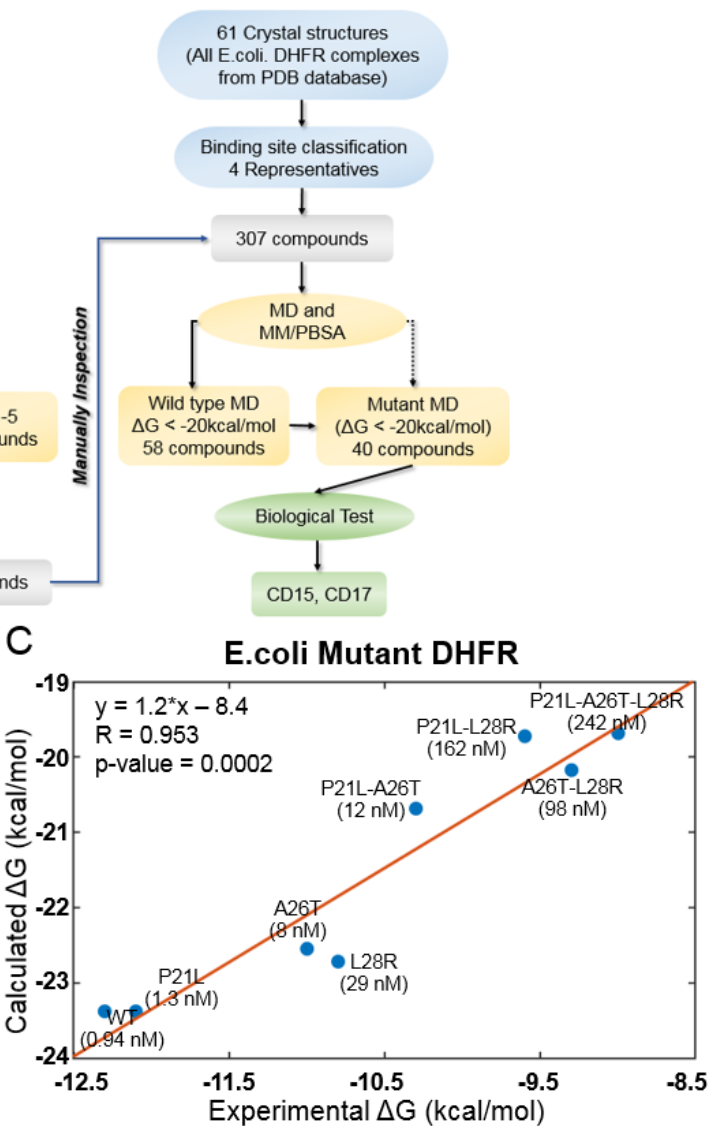

Figure 1 - Computational design of broadly neutralizing DHFR inhibitors effective against WT and resistant DHFR mutant strains. A) Integrative virtual screening workflow. Detailed description of the virtual screening workflow can be found in Method and Materials: Selection of virtual screening hits. B) Linear model for binding affinity prediction constructed using known binding affinities of eight known inhibitors of WT E. coli DHFR (Figure S4) obtained from (Carroll et al., 2012). C) Linear model for binding affinity prediction constructed using experimental inhibitory activity for TMP against WT DHFR and seven resistant DHFR mutants (Rodrigues et al., 2016). MD simulation and MM/PBSA affinity evaluation protocol (Cheron and Shakhnovich, 2017) was applied to calculated binding free energy of complexes of E. coli DHFR with eight known inhibitors and the calculated values were compared with the reported experimental binding affinities (Kd or Ki values).

A total of 307 candidate compounds with strongest docking score that form hydrogen bond with the critical residue Asp27 in the DHFR binding pocket, were submitted for more accurate prediction of binding free energy (Cheron and Shakhnovich, 2017) (Figure 1A). Our approach to predict binding free energy is based on a series of relatively short MD simulations of binding conformations with subsequent MMPBSA scoring as presented in (Cheron and Shakhnovich, 2017). Next, we assessed the accuracy of this approach for WT and mutant DHFR in reproducing binding affinities of known ligands. To that end we built linear regression equation models (see Figure 1B and Figure 1C and Methods and Materials: Construction of binding affinity prediction model) to predict binding free energies calculated by MMPBSA. 
The models reproduced known binding affinities with high accuracy (see Figure $1 \mathrm{~B}, \mathrm{C}$ and Figures S4 and S6). Additionally, we constructed linear regression equation models to predict binding free energy of TMP against WT DHFR from Listeria grayi (L. grayi) and Chlamydia muridarum (C. muridarum) again showing highly significant correlation between predicted and experimental values (Figure S7), demonstrating broad predictive power of the method. More detail on the construction of binding affinity prediction models can be found in the Methods and Materials: Construction of binding affinity prediction model.

Further, the analysis of the DHFR crystal structures showed that Asp27 forms hydrogen bond with almost all DHFR inhibitors in the ligand binding cavity. Thus, compounds predicted by docking that make hydrogen bond with Asp27 (Figure 1A) and having MMPBSA predicted binding free energies less than $-20 \mathrm{kcal} / \mathrm{mol}$ (See Supplementary Materials. Compound Information) against both the WT and all TMP-resistant variants of DHFR were selected for further evaluation. Out of this set, we selected the novel compounds that differ substantially from 183 known DHFR inhibitors (see Method and Materials: Selection of virtual screening hits). Generally, compounds with similar properties tend to have similar activity (Kumar, 2011). Based on that principle, we compared two-dimensional (2D) physicochemical properties (Zhang et al., 2013) and protein-ligand interaction fingerprint (PLIF) features (Marcou and Rognan, 2007) of the prospective set with those of the known inhibitors. Results showed that the selected hits have high similarity in both 2D-physicochemical properties and PLIF features when compared with known DHFR inhibitors, which suggest their potential inhibitory activity against DHFR (Figure S8-S9). On the other hand, they show relatively low chemical similarity (Figure S8) with the known DHFR inhibitors, suggesting that the selected hits are chemically novel. Altogether, a total of 40 prospective active compounds were purchased for evaluation. The detailed information on all compounds can be found in Supplementary Materials (Compound Information). Further details on SBVS can be found in the Method and Materials: Selection of virtual screening hits.

\section{Assaying prospective compounds in vitro}

A spectrophotometric assay (See Methods and Materials) was employed to evaluate possible inhibition of catalytic activity of the 40 selected compounds against WT DHFR and its TMP resistant mutants including P21L, A26T and L28R (Figure 2). All 40 prospective compounds were initially assayed for inhibition of DHFR at a single fixed concentration of $200 \mu \mathrm{M}$. As shown in Figure 2A-2B and Figure S10, a total of 13, 8, 6 and 14 compounds resulted in more than $20 \%$ loss of the DHFR catalytic activity at that concentration for WT, P21L, A26T and L28R DHFR, respectively. Among them, compounds CD15, CD17 and CD08 showed more than $30 \%$ inhibition against both WT and all three DHFR mutants (Figure 2C and Figure S10). Compound CD20, with similar scaffold to that of TMP, showed inhibition against WT, P21L and A26T, but not L28R DHFR and was no longer considered. Thus, a total of three hits including compounds CD15, CD17 and CD08 (Figure 2C) were further evaluated for concentrationdependent inhibition of all DHFR variants. 


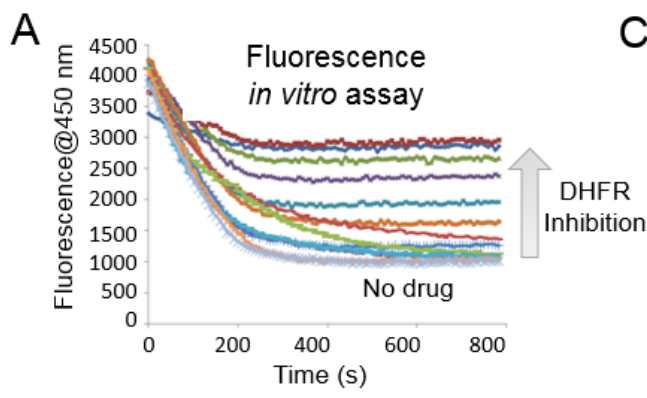

B
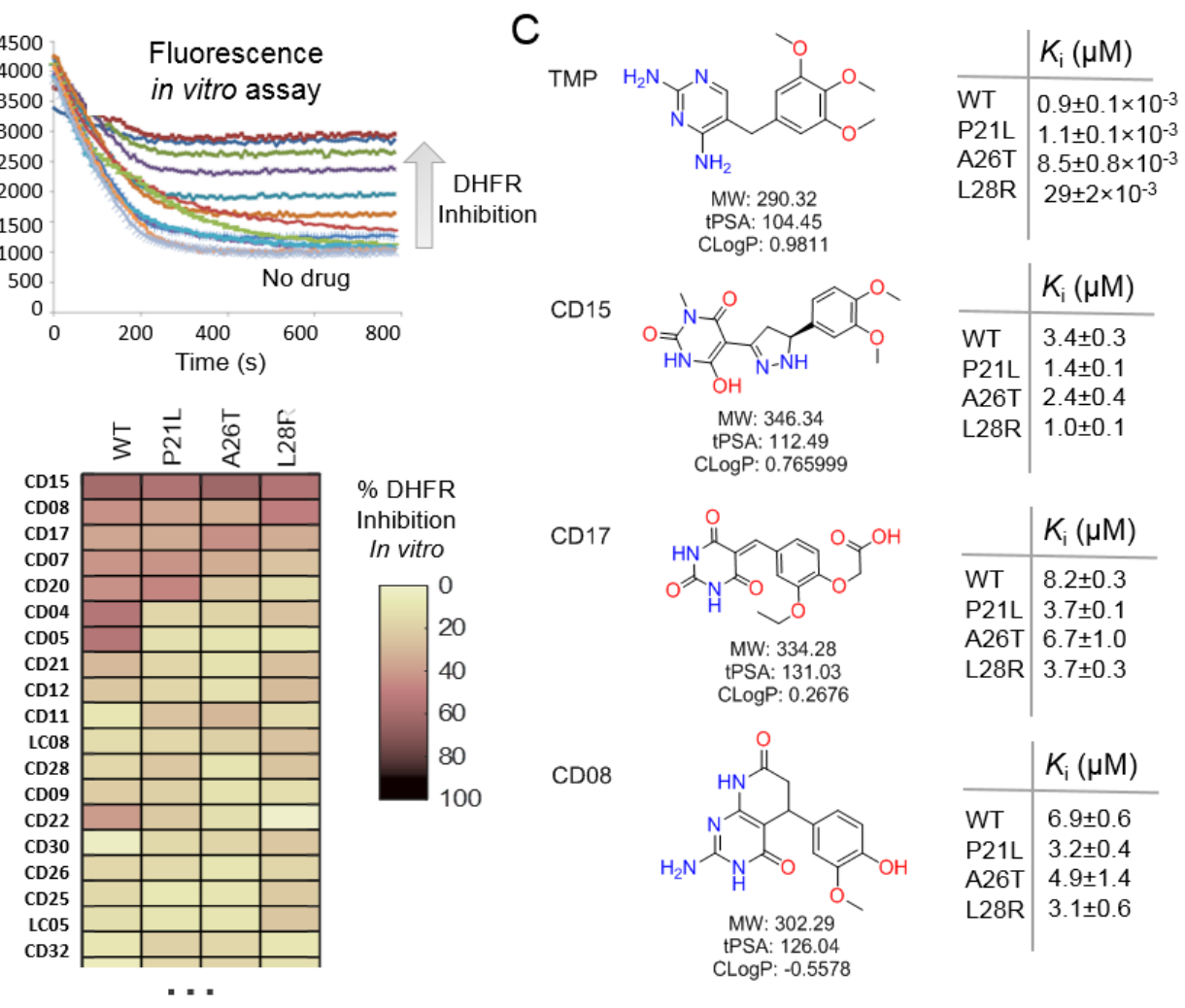

CD17
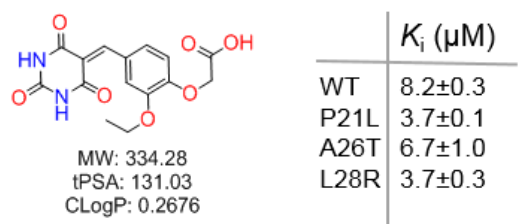

CD08
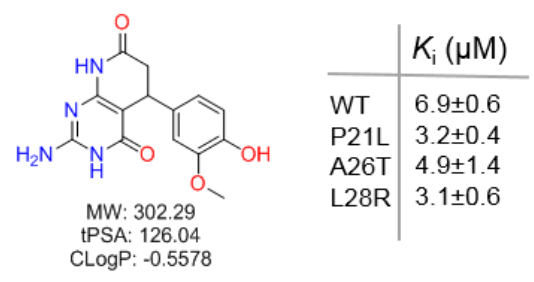

Figure 2-Evaluation of the potential hits in vitro and their optimization. $A-B$ ) An in vitro kinetic assay of DHFR catalytic activity was used to screen inhibitors against WT DHFR and three single mutants resistant to TMP (P21L, A26T and L28R). C) Chemical structures of the top three compounds showing simultaneously the highest potency against WT and mutant DHFR variants. The structure of trimethoprim is shown for comparison.

Two of the three compounds, CD15 and CD17 with two novel scaffolds, inhibited WT and mutant DHFRs in concentration-dependent manner (Figure 3). We used inhibition curves (Figure 3 ), to obtain $\mathrm{IC}_{50}$ values and converted them into corresponding $\mathrm{Ki}$ values (Table 1). For compound $\mathrm{CD} 15$, the Ki values were all less than $5 \mu \mathrm{M}$ against WT and three single-point DHFR mutants. For the L28R mutant the Ki values were $1.0 \mu \mathrm{M}$, outperforming the WT, P21L or A26T mutant DHFR. It is worth noting that L28R is a strong TMP escape variant (Rodrigues et al., 2016; Toprak et al., 2012), thus the discovered CD15 series appeared a promising candidate for an "evolution drug" that has a potential to suppress even most intractable escape variants. Based on these data we decided to proceed with compounds CD15 and CD17 for in depth evaluation. In addition, we evaluated activity of the two most promising inhibitors, CD15 and CD17 against WT DHFR from two more species: L.grayi and C.muridarum. We found that both compounds showed similar inhibition against the two species (See Figure 3 and Table 1). These results suggest the potential of the two compounds as broadly efficient potential antibacterial leads. 

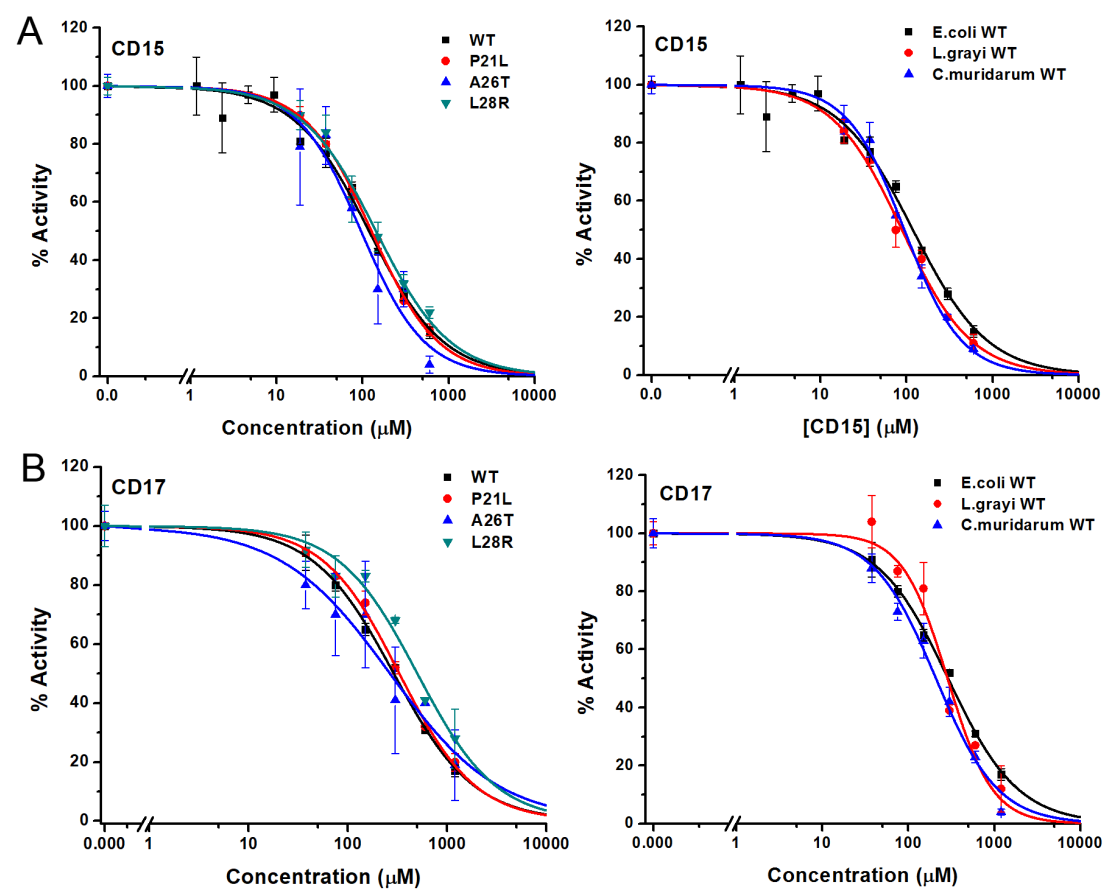

Figure 3-Inhibition of WT and mutant DHFR from different species. by CD15 and CD17. A) Concentration-dependent inhibition curves for compound CD15 for WT and mutant DHFR of E.coli (left panel) and for WT DHFR from E.coli, L.grayi, and C.muridarum, respectively (right panel). B) Concentration-dependent inhibition curves for compound CD17 on WT and mutant DHFR of E.coli (left panel) and of WT DHFR from E.coli, L. grayi, and C. muridarum, respectively (right panel). The \%Activity of the y-axis is represented by the decrease of fluorescence at 450nm for the reaction system (see Methods and Materials for more detail)

We also evaluated inhibitory activity of CD15 against double resistant mutants and found that they are approximately as active or better than against single mutants (see Table 1) 
Table 1 The Ki values (in $\mu \mathrm{M}$ ) for compounds CD15 and CD17.

\begin{tabular}{|c|c|c|c|c|c|c|c|c|c|}
\hline \multirow[b]{2}{*}{ Species } & \multirow{2}{*}{$\begin{array}{c}\text { DHFR } \\
\text { Type }\end{array}$} & \multicolumn{2}{|c|}{ CD15 } & \multicolumn{2}{|c|}{ CD17 } & \multicolumn{2}{|c|}{ CD15-3 } & \multicolumn{2}{|c|}{ TMP } \\
\hline & & $\begin{array}{c}\mathrm{Ki} \\
\text { Value }\end{array}$ & $\mathrm{STD}^{\mathrm{a}}$ & $\begin{array}{c}\mathrm{Ki} \\
\text { Value }\end{array}$ & STD & $\begin{array}{c}\mathrm{Ki} \\
\text { Value }\end{array}$ & STD & $\begin{array}{c}\mathrm{Ki} \\
\text { Value }\end{array}$ & STD \\
\hline \multirow{7}{*}{ E. coli } & WT & 3.35 & 0.28 & 8.18 & 0.29 & 5.52 & 0.98 & $0.90 \times 10^{-3}$ & $0.10 \times 10^{-3}$ \\
\hline & P21L & 1.42 & 0.04 & 3.70 & 0.13 & & & $1.10 \times 10^{-3}$ & $0.10 \times 10^{-3}$ \\
\hline & $\mathrm{A} 26 \mathrm{~T}$ & 2.43 & 0.37 & 6.73 & 1.02 & & & $8.50 \times 10^{-3}$ & $0.80 \times 10^{-3}$ \\
\hline & L28R & 1.04 & 0.07 & 3.65 & 0.33 & 0.98 & 0.11 & $29.0 \times 10^{-3}$ & $2.00 \times 10^{-3}$ \\
\hline & $\begin{array}{l}\text { P21L- } \\
\text { A26T }\end{array}$ & 3.26 & 0.21 & 9.20 & 0.71 & & & & \\
\hline & P21L- & 0.56 & 0.05 & 1.37 & 0.06 & & & & \\
\hline & $\begin{array}{c}\text { A26T- } \\
\text { L28R }\end{array}$ & 0.61 & 0.04 & 2.04 & 0.11 & & & & \\
\hline L. grayi & WT & 5.01 & 0.29 & 16.17 & 1.70 & & & & \\
\hline C. muridarum & WT & 14.60 & 0.64 & 32.38 & 2.53 & & & & \\
\hline Human & WT & 0.38 & 0.05 & 0.74 & 0.10 & $\mathrm{NT}^{\mathrm{b}}$ & & & \\
\hline
\end{tabular}

${ }^{a}$ STD means the standard error from three duplicate experiments.

${ }^{\mathrm{b}} \mathrm{NT}$ means no inhibition was detected at the highest compound concentration tested.

\section{Broad antimicrobial activity of new compounds}

Since two of the 40 compounds inhibit both WT and mutant proteins in vitro, we proceeded to test their efficacy to inhibit growth of E. coli. Previously (Rodrigues et al., 2016) we used strains with chromosomal replacement of WT folA with folA gene encoding three single mutants including P21L, A26T and L28R (Palmer et al., 2015a). All mutant E. coli strains with the chromosomal folA replaced by three drug-resistant variants including P21L, A26T and L28R exhibit elevated resistance to TMP (Rodrigues et al., 2016). In particular, IC50 of TMP for E. coli strain with chromosomal L28R DHFR is about 80 times higher than of WT (Table 2). As shown in Figure 4A and 4B, dose-response curves clearly demonstrated that both CD15 and CD17 inhibit growth of the WT and three single folA mutant E. coli strains. The IC50 values of CD15 and CD17 can be found in Table 2. Importantly, in terms of IC50 CD15 is comparable with TMP on the most resistant variant L28R. These results are consistent with the in vitro enzymatic activity assays (Figure 2C) showing that both CD15 and CD17 inhibited DHFR activity of L28R variant in vitro stronger than WT, accordingly these compounds inhibited growth of the L28R variant stronger than the WT and the other two mutants. 

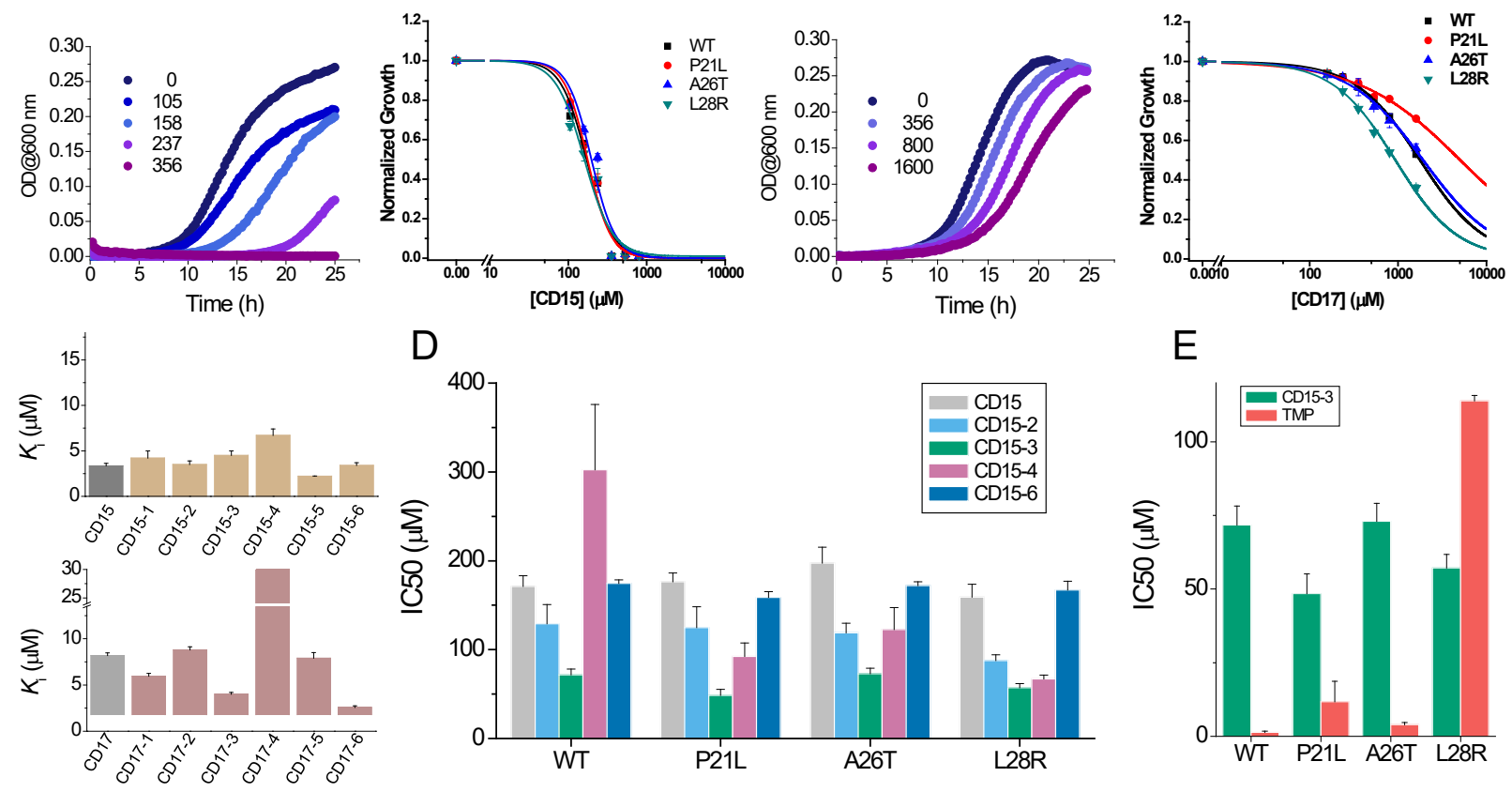

Figure 4. Compounds CD15 and CD17 inhibit growth of WT and TMP-resistant mutant E. coli strains. A) Growth curves for WT strain (left panel) at different concentrations of CD15 and normalized (by maximal growth in the absence of stressor) inhibition by CD15 curves for WT and various TMP resistant DHFR mutants. B) same as A for CD17. Measurements in the presence of different drug concentrations were performed in a 96-well microplate at $37^{\circ} \mathrm{C}$. C) Optimization of compounds CD15 (upper panel) and CD17 (lower panel) lead to hits with increased in vitro inhibitory potency towards WT E. coli DHFR. D) The CD15 series compounds inhibit growth of WT and resistant mutant E. coli strains. For each strain, growth measurements were performed in the presence of varying concentrations of compounds. E) Comparison of IC 50 of inhibition of growth of WT and TMP-resistant mutant E. coli strains for CD15-3 and TMP.

\section{Compound optimization}

While both CD15 and CD17 exhibited desired biological activity, their IC50 for growth inhibition were relatively weak, so we proceeded to optimize both compounds to improve their efficacy. To that end, we searched the ChemDiv database ((http://www.chemdiv.com/) for compounds that are similar to CD15 and CD17. The search yielded a total of 12 extra compounds which were subsequently obtained and evaluated for their inhibitory activity against DHFR in vitro and as inhibitors of $E$. coli bacterial growth. The inhibitory $K_{i}$ values against WT DHFR were in the range of 2 to $8 \mu \mathrm{M}$ for the 6 compounds from the CD15 series and in the range of 3 to $30 \mu \mathrm{M}$ for the 6 compounds from the CD17 series (Figure 4C). Four compounds including CD15-2, CD15-3, CD15-4 and CD15-6 showed better or comparable inhibition for WT and L28R DHFR than CD15 (Figure 4C). Next, we evaluated in vivo activity of these compounds. Results showed that the compound CD15-3 with a naphthalene group (Figure 5A) instead of the trimethoxybenzene of CD15 (Figure 2C) showed marked improvement of efficacy with 3- to 4 times lower IC50 values compared to that of CD15 (Figure 4D). CD15-3 showed 
about 2.4-fold better efficacy than that of TMP on L28R E. coli variant strain (Figure 4E). As potential antibiotic leads are supposed to be selective in targeting bacterial protein and not the host protein we went on to check if CD15-3 interacts and inhibits human DHFR. As shown in table 1 no inhibitory activity for CD15-3 was detected against human DHFR. The drop in the fluorescence signal corresponding to the conversion of NADPH and DHFR mediated formation of THF was persistently observed at all concentrations of CD15-3, indicating no inhibition on human DHFR activity (Figure S11). Structural analysis provided a plausible explanation of selective inhibition of E.coli DHFR by CD15-3 and lack of inhibitory activity against human DHFR (Figure S12). Upon comparison of the crystal structures of E. coli DHFR (PDB 1RX3) and human DHFR (PDB 1KMS), we noted there are significant sequence differences between them in the ligand-binding domain (Figure S12). Different pair of amino acids between E.coli DHFR and human DHFR includes Met20: Leu22, Asp27: Glu30, Leu28: Phe31, Trp30: Tyr33, Ile94: Val115. The lack of inhibition on human DHFR for CD15-3 can be attributed to the loss of the pi-pi interaction with Phe31. As for the E.coli DHFR, the corresponding amino acid is Leu28, and it does not influence much on the binding with CD15-3.

Improved topological polar surface area (tPSA) and clogP of CD15-3 are likely to be responsible for its superior efficacy of the bacterial growth inhibition on WT and mutant strains than other CD15 series compounds (Figure 5A). The $\mathrm{IC}_{50}$ values of growth inhibition are listed in Table 2. Most of the CD17 series compounds did not show significantly better efficacy against L28R than the original CD17 (see Table 2). To address a possibility that CD17 series is a "false positive" mostly targeting another protein(s) we turned to pan assay interference compounds (PAINS) filter that seeks compounds which tend to react non-specifically with numerous biological targets simultaneously rather than specifically affecting one desired target (Baell and Holloway, 2010). Thus, all $12 \mathrm{CD} 15$ and CD17 series compounds were filtered through the PAINS (http://cbligand.org/PAINS/) (Baell and Holloway, 2010). All six CD17 series compounds did not pass the PAINS test and therefore were not considered for further analysis.

Table 2 The $\mathrm{IC}_{50}$ values for the in vivo inhibition of several CD15 and CD17 series compounds

\begin{tabular}{|c|c|c|c|c|c|c|c|c|c|c|c|c|}
\hline \multirow{2}{*}{ DHFR } & \multicolumn{11}{|c|}{ Bacterial growth inhibition $\mathrm{IC}_{50}(\mu \mathrm{M})$} & \multirow[b]{2}{*}{ STD } \\
\hline & $\overline{C D 15}$ & STD $^{\mathrm{a}}$ & CD15-2 & STD & CD15-3 & STD & CD15-4 & STD & CD15-6 & STD & TMP & \\
\hline WT & 170 & 12 & 129 & 22 & 72 & 6 & 302 & 74 & 175 & 4 & 1.3 & 0.4 \\
\hline $\mathrm{P} 21 \mathrm{~L}$ & 176 & 10 & 125 & 24 & 57 & 7 & 92 & 15 & 159 & 6 & 12 & 2 \\
\hline A26T & 197 & 18 & 119 & 11 & 73 & 6 & 123 & 25 & 172 & 4 & 3.9 & 0.7 \\
\hline L28R & 159 & 14 & 88 & 6 & 48 & 5 & 67 & 4 & 167 & 10 & 114 & 7 \\
\hline \multirow{2}{*}{ DHFR } & \multicolumn{11}{|c|}{ Bacterial growth inhibition $\mathrm{IC}_{50}(\mu \mathrm{M})$} & \\
\hline & 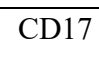 & STD & CD17-3 & STD & CD17-4 & STD & & & & & & \\
\hline WT & 1774 & 22 & 2946 & 967 & $\mathrm{ND}^{\mathrm{b}}$ & ND & & & & & & \\
\hline $\mathrm{P} 21 \mathrm{~L}$ & 5048 & 809 & 2845 & 437 & ND & ND & & & & & & \\
\hline A26T & 1920 & 135 & 2152 & 265 & ND & ND & & & & & & \\
\hline L28R & 932 & 44 & 907 & 57 & 1857 & 272 & & & & & & \\
\hline
\end{tabular}


${ }^{a} S T D$ means the standard error from three duplicate experiments.

${ }^{\mathrm{b}} \mathrm{ND}$ (not determined) indicates no result was obtained for the compound against WT and three mutant DHFR.

The binding mode of the two most promising compounds CD15 and CD15-3 were evaluated using molecular docking (Figure 1a, Glide XP mode) with the target E. coli DHFR (PDB 1RX3). As shown in Figure 5B and 5C, both compounds formed two hydrogen bonds with the key residue Asp27 by the hydroxy group in the 6-hydroxy-3-methylpyrimidine-2,4 $(1 \mathrm{H}, 3 \mathrm{H})$-dione scaffold. The binding modes of CD15 and CD15-3 overlapped perfectly with the binding conformation of TMP, providing rationale for the inhibitory activity of CD15 series. However, in addition to the hydrogen bond formed with Asp27, TMP also forms hydrogen bond with another critical residue Ile94 as well as the conserved water molecule HOH302. Nevertheless, unlike TMP, our hit compounds showed broad inhibitory activity in vitro and in vivo on both the WT and mutant DHFR strains. The broad activity of CD15 compounds can be explained, in part by a dihydro-1H-pyrazole group in the same position as methylene group of TMP. Naphthalene group of CD15-3 extends further in DHFR binding pocket than the corresponding trimethoxybenzene of TMP, potentially resulting in additional hydrophobic interaction with L28R in DHFR which provides structural rationale for strong potency of CD15-3 against resistant L28R variant.

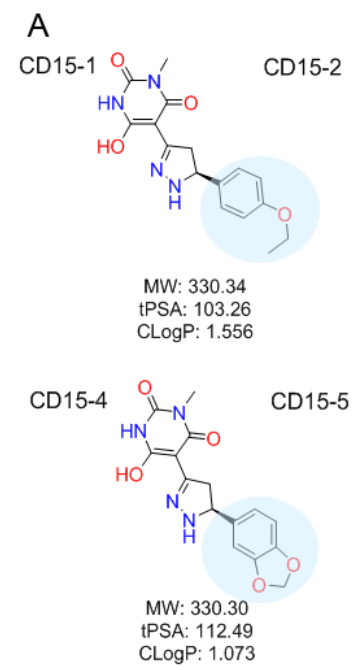

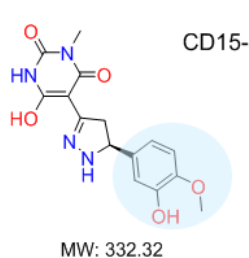

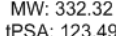
CLogP: 0.2902

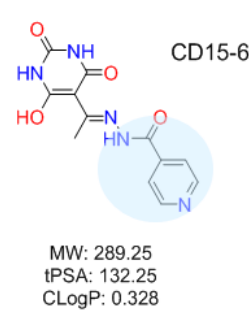

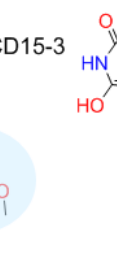

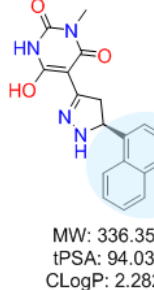

CLogP: 2.282

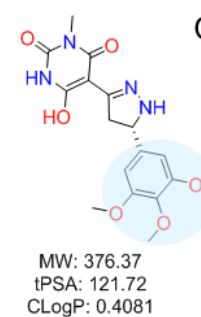

B

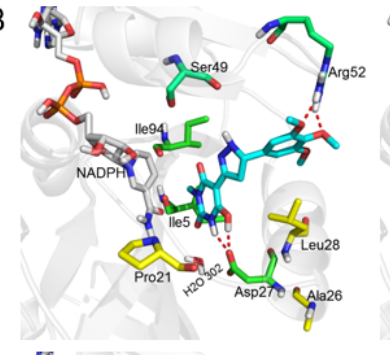

C

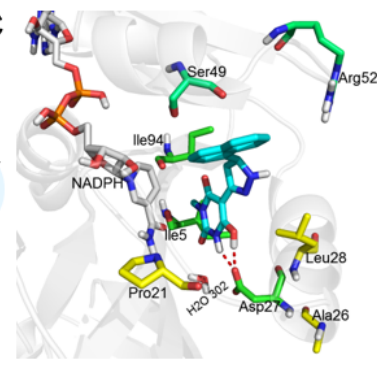

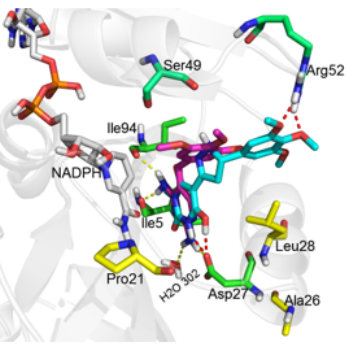

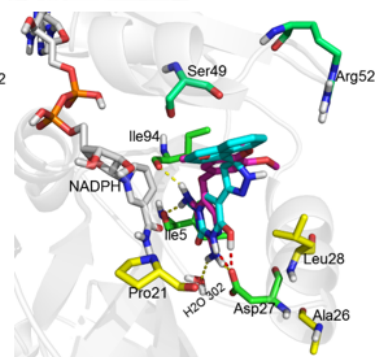

Figure 5 Optimization of the compounds of CD15 series A) Chemical structures of $2^{\text {nd }}$ generation variants of compound CD15 selected for further experimental testing. B) The binding interaction of CD15 with DHFR (left panel) and the alignment of CD15 (cyan stick) with TMP (purple stick) in the binding pocket (right panel). C) The binding interaction of CD15-3 with DHFR (left panel) and the alignment of CD15-3 (cyan stick) with TMP (purple stick) in the binding pocket (right panel).

\section{Target validation in vivo}

To confirm DHFR as the intracellular target of CD15-3 we overexpressed DHFR in E. coli cells to assess whether it rescues growth inhibition by CD15-3. To that end we transformed E. coli 
BW27783 with pBAD plasmid (empty plasmid for the control and with folA gene for DHFR expression). BW27783 cells constitutively express arabinose transporters providing rather homogeneous response from the cell pool under arabinose induction (Bhattacharyya et al., 2016). Interestingly controlled expression of folA (encoding DHFR) under pBAD promoter with $0.005 \%$ arabinose induction partially rescued growth in a certain range of CD15-3 concentrations (Figure.6A). This improvement of growth rate was less pronounced at higher concentration of CD15-3. For control we used WT cells transformed with empty pBAD plasmids (without folA gene) and observed no effect on growth.

To further probe whether CD15-3 inhibits intracellular DHFR we overexpressed an inactive variant of DHFR, D27F mutant using the same pBAD-promoter where the expression was induced by $0.005 \%$ external arabinose (Rodrigues and Shakhnovich, 2019; Tian et al., 2015). In this experiment overexpression of the $\mathrm{D} 27 \mathrm{~F}$ mutant did not rescue growth in cells treated with CD15-3. Inhibition by CD15-3 of cells overexpressing D27F mutant was almost identical to that of WT at all concentrations of CD15-3.. (Figure 6B). This result showed that inhibition of DHFR in vivo was, at least partially, responsible for inhibition of cellular growth induced by CD15-3. As TMP is a known inhibitor of DHFR (WT) we wanted to check if these overexpression plasmid systems behave in a similar way as in the case of CD15-3 inhibition. Similar trend was observed for cells treated with TMP. Overexpression of WT DHFR rescued growth of TMP treated cells (Figure S13) while no rescue of growth from TMP induced inhibition was observed upon overexpression of inactive D27F variant (Figure S13). These results indicate that DHFR is an intracellular target for the new compound CD15-3. However, a possibility remains that CD15-3 does not target DHFR exclusively. To understand if CD15-3 is targeting cellular DHFR and thereby disrupting folA pathway, we further performed growth experiments in the presence of supplement folA mix. folA mix which is comprised of purine, thymine, glycine and methionine functions as a metabolic supplement for cells with diminished DHFR function (Singer et al., 1985). E.coli cells were grown in M9 media supplemented with "folA mix" in presence and absence of CD15-3. We found that growth of CD15-3 treated cells was partially rescued by folA mix and again the effect was more prominent at relatively lower concentrations of CD15-3 (Figure 6C).

At the same time, we observed only partial rescue of CD15-3 inhibited growth by folA mix or DHFR complementation at higher concentrations of CD15-3 suggesting that at high concentrations this compound might inhibit other proteins besides DHFR. In the follow up publication (Chowdhury et al., 2021) we used system-level approaches to discover additional target of CD15-3, besides DHFR. 

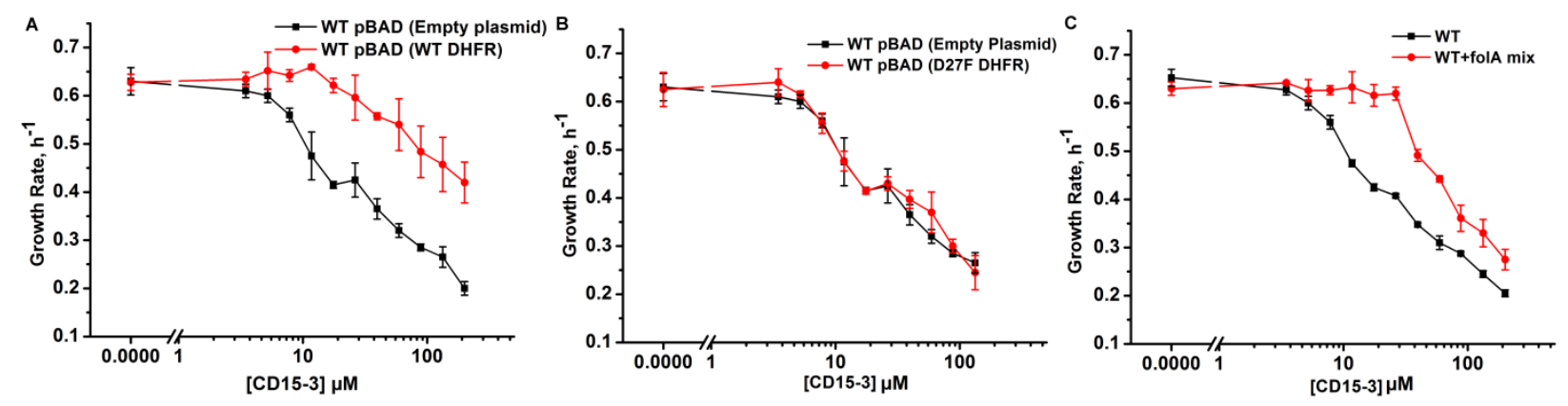

Figure 6 Overexpression of functional (WT) DHFR shows partial recovery from CD15-3 induced growth inhibition. (A) Overexpression of WT DHFR using pBAD-promoter and at $0.005 \%$ arabinose induction showed improvement in growth rates compared to cells with empty pBAD-plasmid (lacking DHFR gene) under conditions of CD15-3 treatment. (B) Comparative growth rate profiles of $W T$ (with empty $p B A D$-plasmid) and $W T$ overexpressing $D 27 F$ inactive mutant of DHFR. The growth rate profiles clearly show that D27F mutant of DHFR could not rescue cells from CD15-3 induced growth inhibition. (C) Comparative growth rate profiles of cells grown in presence of folA mix supplement under conditions of CD15-3 treatment. Cells grown in presence of folA mix metabolic supplementation showed partial rescue in growth under conditions of CD15-3 treatment. 
bioRxiv preprint doi: https://doi.org/10.1101/2020.10.30.362582; this version posted May 25, 2021. The copyright holder for this preprint (which

was not certified by peer review) is the author/funder, who has granted bioRxiv a license to display the preprint in perpetuity. It is made available under aCC-BY-NC-ND 4.0 International license.

CD15-3 largely prevents evolution of resistance in $E$. coli. 


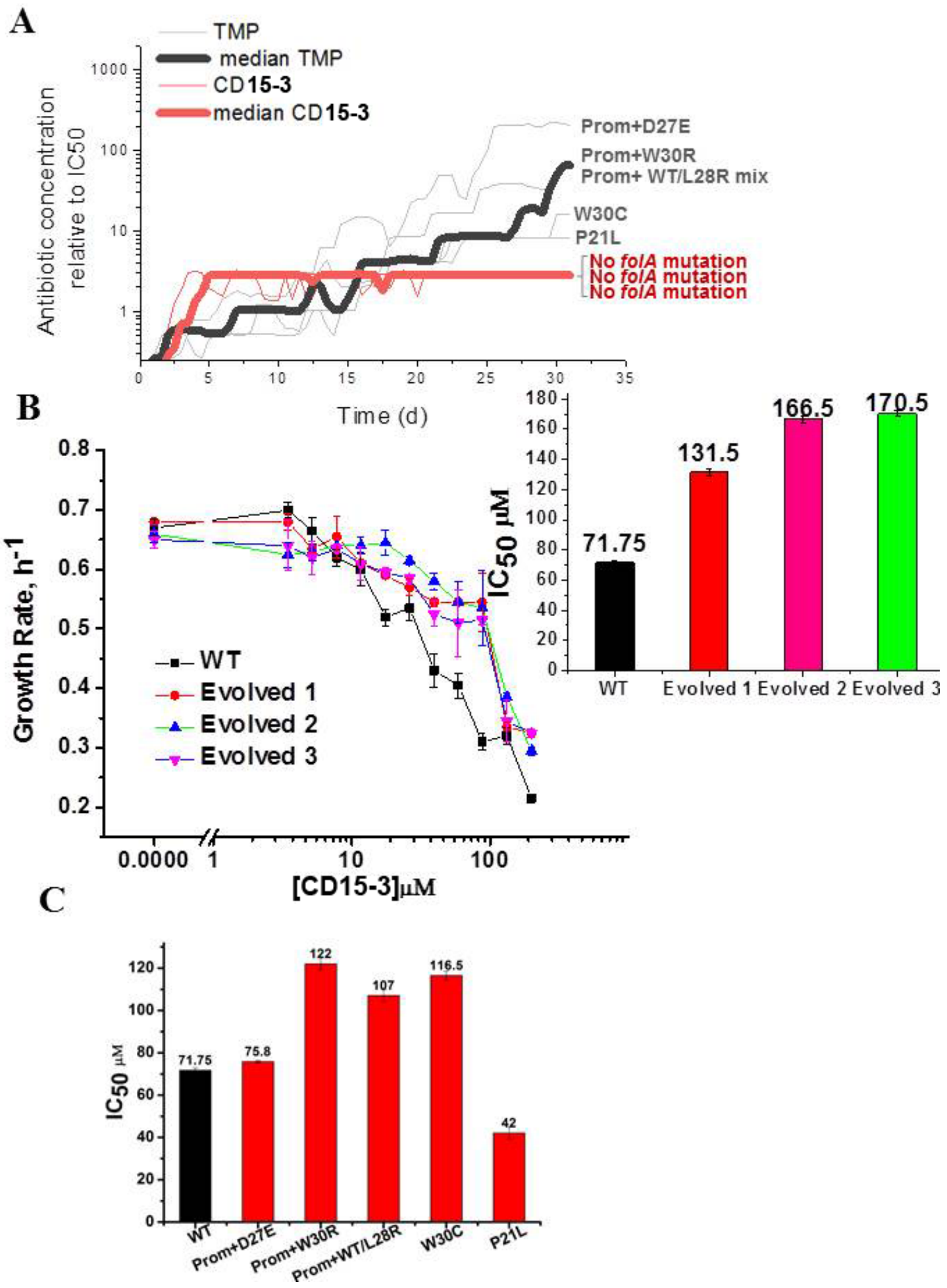

Figure 7. Resistance to CD15-3 evolves slowly. (A) Grey and red traces show evolution of antibiotic resistance against TMP and CD15-3 respectively. Red (bold) and black (bold) traces shows the median traces of 3 replicate evolutionary trajectories under selection pressure from CD15-3 and TMP respectively. Under pressure from TMP cells evolved TMP resistance with IC 50 between 8 to 200 fold higher than original naïve E. coli strain. Cells evolved under CD15-3 
treatment showed IC50 values for CD15-3 between 131.5 $\mathrm{MM}$ to $170.5 \mu \mathrm{M}$ which is about 2-fold higher in comparison to IC50 for naïve WT. The antibiotic concentrations represented were obtained for a single evolutionary trajectory and are normalized to IC50 of naïve cells to TMP and CD15-3 (1.3 $\mu M$ and $71.75 \mu M$ respectively). Cells which evolved under TMP treatment (TMP escape) in individual evolution trajectories showed D27E, W30R, W30C and P21L mutations in the folA locus along with several other mutations outside of folA. One of the TMP trajectories had a mix of WT and L28R population along with promoter mutation. No mutation in the folA locus was observed in the CD15-3 evolved cells. (B) Growth rates in WT and evolved strain in a range of CD15-3 concentrations showing weak resistance of evolved strains. Inset shows the differences in IC 50 values in WT and evolved forms (from three evolution trajectories). (C) CD15-3 also inhibits the growth of the TMP escape mutant. D27E escape mutant had similar IC50 to CD15-3 as is observed with the naïve/WT. IC50 for CD15-3 obtained for W30R, W30C and WT/L28R mix set was found to be less than two fold higher than the WT.

The primary focus of our approach to design evolution drugs that block pathogen's escape routes, is on the search for inhibitors of a single protein target which would be equally effective against WT and resistant variants,. To determine how fast WT E. coli can acquire resistance to CD15-3, we evolved three trajectories of $E$. coli under continuous exposure to the drug for over one month. We used a previously described automated serial passage protocol (Rodrigues and Shakhnovich, 2019) that adjusts the drug concentration in response to increase of growth rate caused by emergent resistant mutations thus maintaining evolutionary pressure to escape antibiotic stressor (see Methods and Materials section for details). We found that, at early stages of evolution, the CD15-3 concentration necessary to reduce the growth rate of the culture to $50 \%$ (with respect to non-inhibited naïve cells) increased to a value about 2.7 times higher than the IC 50 of the naïve strain. However, at subsequent time points, the CD15-3 concentration remained constant, indicating that the cells were unable to further develop resistance to CD15-3. We found no mutations in the folA locus of the evolved strains upon Sanger sequencing, indicating that the modest increase in resistance to CD15-3 is not associated with target modification. In parallel, we also studied evolution of resistance to TMP in 5 independent trajectories using the same approach. At the end of the evolutionary experiment, the cells evolved TMP resistance with $\mathrm{IC}_{50}$ between 8 to 200 fold higher than original naïve E. coli strain (Figure 7A). Sanger sequencing of the folA locus revealed point mutations in the folA locus in all trajectories. We found mutations in the DHFR active site, which are associated with resistance to TMP (Oz et al., 2014) and also in the promoter region, which are known to increase expression of DHFR (Tamer et al., 2019)

To verify the results of evolutionary experiment, the population of cells evolved in presence of CD15-3 was further plated and two colonies were isolated. We measured growth of evolved variant of $E$. coli in M9 media using the same concentration range of CD15-3 as was used for WT cells. Evolved strains exhibited $\mathrm{IC}_{50}$ for $\mathrm{CD} 15-3$ in the range of $131.5 \mu \mathrm{M}$ to $170.5 \mu \mathrm{M}$, about 2-fold higher compared to $\mathrm{IC}_{50}$ for WT (Figure 7B). In the other evolution trajectories with TMP selection escape mutants which emerged were W30R, W30C and a culture which was a mixed population of WT cells (with promoter mutation) and cells with L28R folA mutation. IC 50 values obtained for CD15-3 against TMP escape W30R and W30C were respectively 1.7 and 1.6 times higher compared to naïve/WT cells. The TMP trajectory which had a mix of WT cells with 
promoter mutation and L28R folA mutation had an $\mathrm{IC}_{50}$ for $\mathrm{CD} 15-3$ which was 1.5 times higher than naïve/WT cells

It is to be noted that CD15-3 was designed to inhibit a subset of TMP escape mutants viz. P21L, L28R and A26T. Interestingly CD15-3 inhibited the D27E TMP escape mutants along with P21L mutant with $\mathrm{IC}_{50}$ close to naïve WT strain (Figure 7C). Higher $\mathrm{IC}_{50}$ against W30 mutants could potentially mean reduced inhibitory interaction for CD15-3 against these mutants. Further in all these escape mutants there could be mutations outside folA locus and hence could not be captured by Sangers sequencing of the folA locus. Still $\mathrm{IC}_{50}$ for these TMP escape mutants against CD15-3 is less than two fold higher compared to that of WT.

Further, we carried out a complementary plate-based "one-shot" selection experiment to determine if selection under CD15-3 stress leads to the emergence of colonies with mutations in the folA locus. WT naïve cells were grown in M9 media without antibiotic to allow standing variation in the growing culture. At $10^{3}$-fold dilution of a culture that has grown for 8 hours, cells were plated on M9 agar plates with either CD15-3 or TMP added. CD15-3 and TMP concentrations were kept 3 times the respective IC50 values (w.r.t. WT i.e. $210 \mu \mathrm{M} / \mu \mathrm{l}$ CD15-3 and $1.2 \mu \mathrm{g} / \mu \mathrm{TMP}$ ). A few isolated colonies were found under these conditions. We sequences folA locus in six isolated colonies from each of CD15-3 and TMP plates and their IC50 values were measured. None of the colonies that grew on CD15-3 plates showed mutation in the folA locus (Table 3)., while 4 of the six colonies isolated from the TMP plate showed mutations in the folA locus. The colonies formed on the CD15-3 plate had $\mathrm{IC}_{50}$ values roughly 2 to 2.3 times higher than against WT (Table 3). On the other hand the colonies which formed on the TMP plate had much higher $\mathrm{IC}_{50}$ values compared to TMP against WT (typically around $1.3 \mu \mathrm{M}$ ).

In addition to our in vitro evolution study, the plate based study shows further that CD15-3 escape route does not involve mutation in the folA locus and the CD15-3 resistant forms which emerge upon CD15-3 selection are marginally more resistant compared to the naïve/WT cells

Table 3 folA locus mutation and IC50 values of TMP and CD15-3 resistant colonies which appeared on the TMP and CD15-3 plates

\begin{tabular}{llllllll}
\hline $\begin{array}{l}\text { Colony } \\
\text { No }\end{array}$ & Selection & $\begin{array}{l}\text { folA } \\
\text { mutation }\end{array}$ & $\begin{array}{l}\text { Mean } \\
\text { IC50 fold } \\
\text { increase } \\
\text { (w.r.t. } \\
\text { WT) }\end{array}$ & $\begin{array}{l}\text { Colony } \\
\text { No }\end{array}$ & Selection & $\begin{array}{l}\text { folA } \\
\text { mutation }\end{array}$ & $\begin{array}{l}\text { Mean } \\
\text { IC50 fold } \\
\text { increase } \\
\text { (w.r.t. } \\
\text { WT) }\end{array}$ \\
\hline 1 & & 100 & 1 & CD15-3 & no & 1.9 \\
2 & TMP & L28R & 110 & 2 & CD15-3 & no & 1.8 \\
3 & TMP & L28R & 110 & CD15-3 & no & 2 \\
4 & TMP & M20I & 7 & 3 & CD15-3 & no & 2.3 \\
5 & TMP & no & 10.6 & 4 & CD15-3 & no & 2.3 \\
6 & TMP & W30C & 23.3 & 5 & CD15-3 & no & 2.2 \\
\hline
\end{tabular}




\section{Whole Genome sequencing of the evolved variant}

We performed whole genome sequencing of the strains evolved under CD15-3 using two isolated colonies (mentioned as E1 and E2 in Figure. 8A) obtained from our evolution trajectories keeping naïve BW25113 strain as a reference. No mutations at or upstream of the folA locus was found. Therefore, the developed moderate resistance against CD15-3 could not be attributed to target modification.

Further analysis of the sequencing results revealed regions of duplication in the genome of the evolved strain as observed by the double depth-height (Figure.8A). Depth or coverage in sequencing outputs refer to the number of unique reads that include a given nucleotide in the sequence. The duplicated segment was found to be a stretch of above $81 \mathrm{~KB}$. In the context of evolution of antibiotic resistance, the relatively frequent occurrence of genome duplications by amplification suggests that evolution of gene dosage can be a faster and more efficient mechanism of adaptation than rare downstream point mutations (Sandegren and Andersson, 2009). The gene that confers the limitation is often amplified in this mechanism, however, sometimes increased dosage of an unrelated non-cognate gene can resolve the problem.

Multiple genes belong to the region of genome duplication in the evolved strain (Figure. 8B) including transporter and efflux pump genes, transposable elements, stress response genes and metabolic genes viz. oxidoreductases, dehydrogenases, kinase regulators etc. The duplicated segment in the genome of the evolved variant contained genes encoding porin proteins, $\mathrm{ABC}$ transporter permeases and cation efflux pump genes (cus-genes). The CusCFBA system is a HME (heavy metal efflux)-RND system identified in E. coli. Resistance-nodulation-division (RND) family transporters refer to a category of bacterial efflux pumps primarily observed in Gram-negative bacteria. They are located in the membrane and actively transport substrates. Cus-efflux system was initially identified for the extrusion of silver $(\mathrm{Ag}+)$ and copper $(\mathrm{Cu}+)$. They have been found to induce resistance to fosfomycin (Nishino and Yamaguchi, 2001), dinitrophenol, dinitrobenzene, and ethionamide (Coutinho et al., 2010). The set of genes constituting cus-system viz. cusCFBA, are all located in the same operon (Gudipaty et al., 2012). The system is composed of the RND efflux pump (CusA); the membrane fusion protein, MFP (CusB); and of the outer membrane protein, OMP (CusC). The assembly of these proteins have been reported to be identical to the AcrB, $\mathrm{CusA}(3)$ : $\mathrm{CusB}(6)$ : $\mathrm{CusC}(3)$ (Delmar et al., 2013). In the duplicated segment of the evolved variant the entire cus-efflux system was found to be present.

We carried out the metabolic characterization of strains evolved in the presence of CD15-3 and naïve strains by LC-MS to further investigate the mechanism of resistance to the drug (a detailed analysis is in a subsequent publication) (Chowdhury et al., 2021). Interestingly, we found markedly lower abundance of CD15-3 in the evolved strain compared to WT cells suggesting a possible efflux pump mediated compound depletion (Figure.8C). After four hours of CD15-3 treatment the abundance of the drug was found to be around 10 percent of the initial abundance. Drug efflux is a key mechanism of resistance in Gram-negative bacteria (Masi et al., 2017; Sandegren and Andersson, 2009). Pumping out drug compound under conditions of drug 
treatment is probably the most direct and nonspecific way of combating the toxic effect of a drug. It is interesting to note that we observed higher $\mathrm{IC}_{50}$ in the evolved strain compared to the naïve strain for some other antibiotics which we tested. Both the naïve and CD15-3 evolved cells were treated with TMP and Sulphamethoxazole. Sulphamethoxazole a sulfanilamide, is a structural analog of para-aminobenzoic acid (PABA) and binds to dihydropteroate synthetase. Under both treatment conditions CD15-3 evolved cells partially escaped the drug inhibition and showed about 3-fold higher IC50 for TMP and Sulphamethoxazole (Figure 8D and E). These results show that the efflux mediated drug resistance in the evolved strain is non-specific. It demonstrates a potential strategy for antibiotic cross resistance and helps bacteria to escape inhibitory actions of CD15-3 and other antibacterial compounds with completely different protein targets. In the same vein we note that the efflux pump mechanism shows only moderate increase of IC50 for a variety of antibiotics in contrast to about 200-fold increase in several strains evolved under TMP (Fig.7). 
A

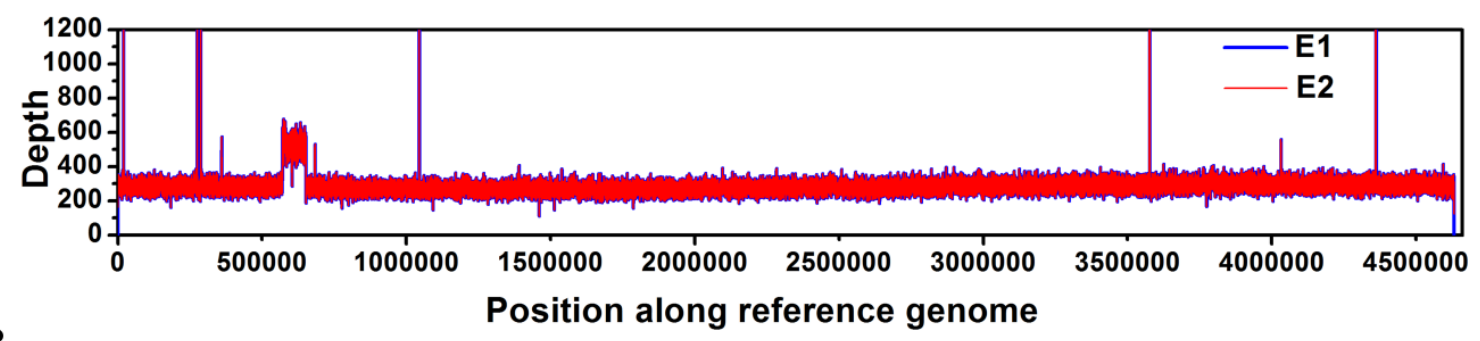

B
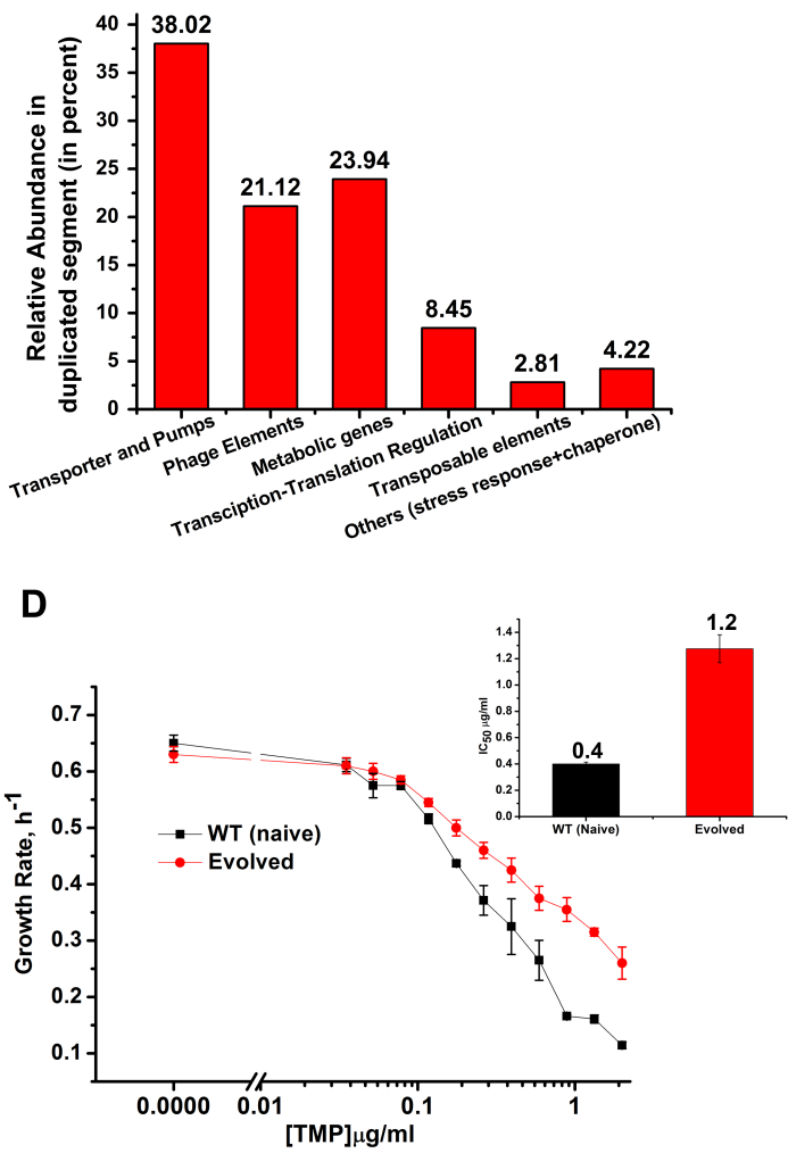

C
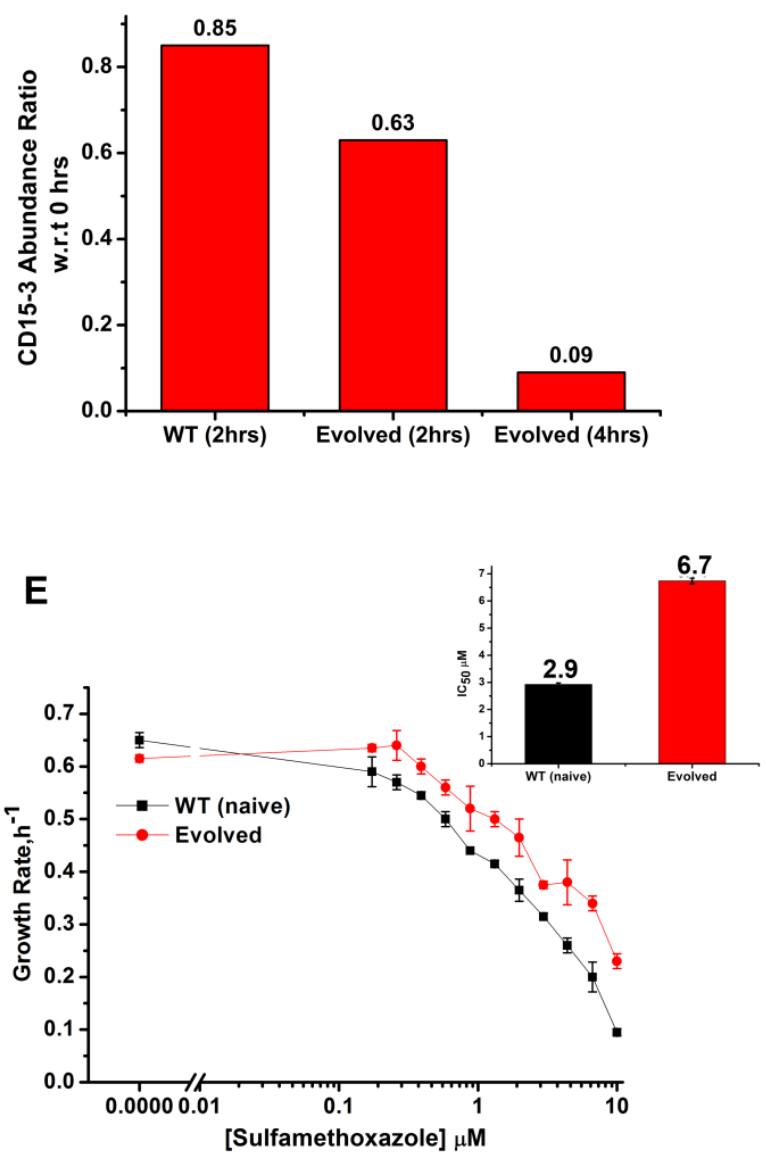

Figure 8. Whole genome sequence of evolved variant revealed region of genome duplication. (A) Whole genome sequence display of the evolved form on alignment to BW25113 reference genome. The display shows regions of duplication as observed by increased height as per depth axis. (B) Bar plot showing the relative abundance of genes which constitutes duplicate segment in the genome of evolved form. (C) Bar plot showing the relative intracellular concentration of CD15-3 (with respect to the intracellular concentration in naïve cells at zero hour) in naive and evolved strains at various time points of treatment. (D) CD15-3 evolved cells show cross resistance to other antibiotics. Growth rate profiles of WT (naïve) and CD15-3 evolved cells grown under varying concentrations of trimethoprim (TMP). CD15-3 evolved cells grow better 
under TMP treatment and have almost 3 fold higher IC50 (shown in inset) compared to WT (naive). (E) Growth rate profiles of WT (naive) and CD15-3 evolved cells grown in presence of Sulfamethoxazole shows CD15-3 evolved cells grow better compared to WT (naive) cells (as reflected by the growth rates). CD15-3 evolved cells show somewhat higher IC50 compared to WT (naive) when grown in presence of Sulfamethoxazole (shown in in inset).

\section{Morphological changes induced by CD15-3 treatment.}

As cellular filamentation and concomitant morphological changes are one of the visible hallmarks of stress responses to inhibition of proteins on the folate pathway of which DHFR is a member,(Ahmad et al., 1998; Justice et al., 2008; Sangurdekar et al., 2011; Zaritsky et al., 2006) we proceeded to image $E$. coli cells in absence of CD15-3 (control) and with added CD15-3. . Experiments were performed at 42 degrees Celsius. Cells were grown for 4 hours before image acquisition. DIC imaging results of the CD15-3 treated WT cells (Figure 9B) showed filamentation as compared to the cells grown in absence of CD15-3 (Figure 9A). Treated cells showed a wide distribution of cell length with a median length which was more than double than that of the untreated sets (Figure 9E).

Similar imaging experiment was also carried out for the CD15-3 resistant variant of $E$. coli cells obtained in our evolution experiment. These cells (Figure 9C) had similar median cell lengths as WT (naïve) cells before CD15-3 treatment. Upon CD15-3 treatment these evolved cells showed slightly higher median cell length, although no visible filamentation was observed (Figure 9D). As efflux genes comprise $38 \%$ of the duplicated genome segment and CD15-3 abundance after 4 hours of treatment was markedly lower (compared to naive WT) an efflux mediated drug resistance mechanism could potentially explain as to why evolved cells did not show any CD153 induced stressed morphology. The slight increase in median cell length in evolved cells upon treatment could be attributed to the fact that the transporter and efflux pump mediated resistance is a rather generic way to escape drug action and is limited in its ability to completely evade drug action. 


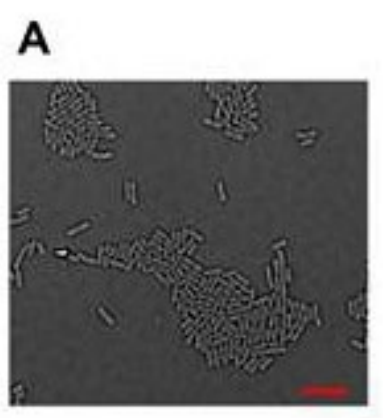

B

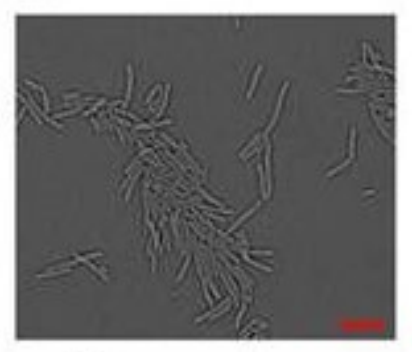

E
C
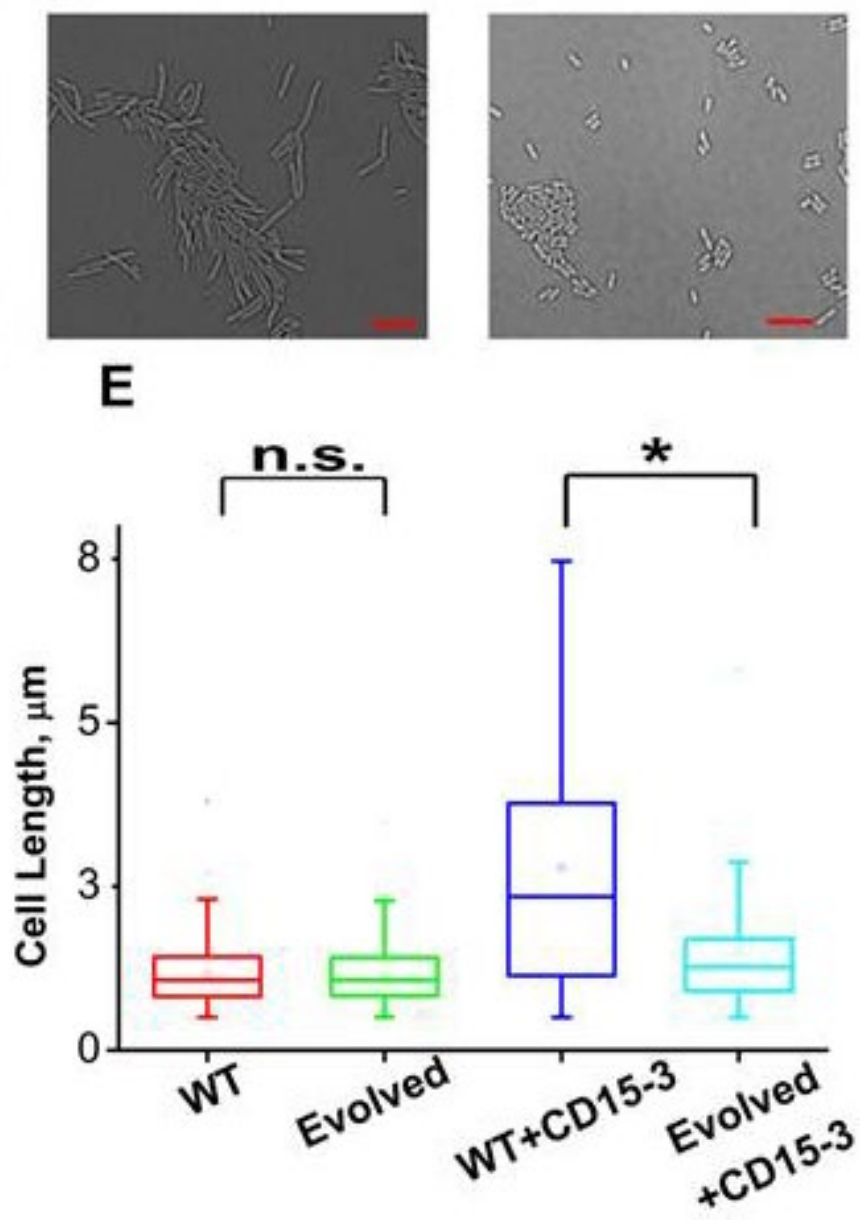

D

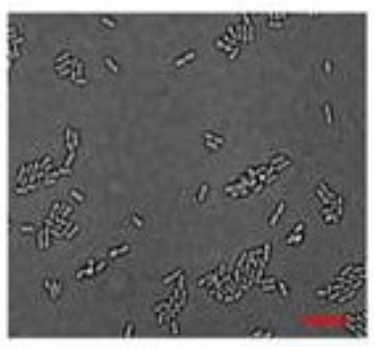

Figure 9. CD15-3 treatment leads to stress induced morphological changes in WT $E$. coli cells. DIC image of WT cells under (A) control (no CD15-3 treatment) and (B) treated (CD15-3 treatment) conditions. (C) DIC image of evolved E. coli cells under control (no CD15-3 treatment) and (D) CD15-3 treated condition. (E) Distribution of cell lengths as derived from DIC imaging of WT and evolved cells under control and CD15-3 treatment conditions. Scale bar corresponds to a cell length of $2 \mu \mathrm{m}$. Untreated WT (naive) and evolved cells had comparable cell lengths with median cell lengths of $1.07 \mu m$. (n.s. indicates the distribution of cell lengths were not significantly different, Mann-Whitney test, $p$-value $=0.961$ ). Both WT and evolved cells were subjected to CD15-3 treatment at IC50 concentrations. WT treated cells were observed to have a filamentous morphology and the median cell length $(2.34 \mu \mathrm{m}$.$) double of that of the$ untreated WT set. Evolved cells after CD15-3 treatment had a median cell length of 1.26_um which is slightly higher than that of untreated set. But the cell size distribution of the evolved cells showed much less change after CD15-3 treatment compared to that observed for the WT (* indicates the distribution of cell lengths were significantly different, Mann-Whitney test, p-value $<0.001)$. 


\section{Discussion}

Antibiotic resistance has emerged as a critical threat in the treatment of infectious diseases. This challenge is primarily an evolutionary problem which nails down to factors like antibiotic induced selection, the population structure of the evolving microbes and their genetics of adaptation (Roemhild and Schulenburg, 2019). Horizontal gene transfer - another critical mechanism conferring antibiotic resistance (Dzidic and Bedeković, 2003) in bacterial population - presents further complications . Traditional antibiotic design protocols and development pipelines do not essentially consider this high intrinsic potential of bacterial adaptation in antibiotic stressed growth conditions and hence do not properly address the problems associated with drug resistance. This problem has become more acute with the emergence of multidrug resistant bacteria which can potentially escape the actions of a wide array of antibiotic formulations and have a complex landscape of evolutionary adaptation.

To tackle these problems, complex treatment strategies for infectious diseases like combinationdrug therapies or sequential drug therapies have been developed (Baym et al., 2016; Vestergaard et al., 2016). These strategies are considered to be superior to classical monotherapies as instead of one protein target they aim at multiple molecular targets. It is interesting to note that natural product antibiotics like penicillin and other beta lactam drugs are inherently multi-target antibiotics (Tyers and Wright, 2019). Same applies to tetracyclin and aminoglycosides which targets bacterial ribosome and in turn interact with multiple ribosomal proteins (Forge and Schacht, 2000). All these prompted interest in targeting multiple molecular targets with an aim to suppress the emergence of antibiotic resistance by target modification (Silver, 2007).

However, in many cases these strategies enjoyed only limited success in constraining the emergence of evolutionary escape variants and rare multidrug variants (Hegreness et al., 2008). Furthermore, none of these strategies act proactively by suppressing future evolutionary escape variants and remain more as a monotherapy in sequence or a combination therapy targeting WT variants.

Evolution drugs were envisioned as chemical formulations in the potential anti-evolution strategy which would prevent or delay evolution of drug resistance. Recent studies highlighted the bacterial evolutionary landscape under antibiotic induced selection pressure and the potential drug escape routes (Palmer et al., 2015b) (Cao et al., 2017; Ogbunugafor et al., 2016; Rodrigues et al., 2016). In a parallel development the evolutionary dynamics of viral escape from drug or antibody stress was studied in silico and in vivo (Doud et al., 2018; Gaebler et al., 2021; Louie et al., 2018; Marchi et al., 2019; Rotem et al., 2018) This knowledge is critical to design better strategies to block the emergence of drug or antibody resistant phenotypes of pathogens.

One of the best-studied examples is evolving resistance to inhibitors of DHFR. Rapid clinical resistance to available antifolates like TMP emerges merely after three rounds of directed evolution and sequential fixation of mutations through ordered pathways has been shown to contribute to the evolutionary paths for antibiotic resistance (Tamer et al., 2019; Toprak et al., 2012). Even though several classes of small molecules have been investigated for their potential 
antifolate activity, the rapid emergence of resistance by readily accessible mutational pathways in the folA gene pose an immense challenge (Toprak et al., 2012). Several studies have shown that $E$. coli cells evolved in the presence of trimethoprim, a DHFR inhibitor, can become virtually insensitive to the drug upon successive accumulation of mutations in target gene. It is thus urgent to develop new tools for the systematic identification of novel scaffolds and discovery of inhibitors that interact simultaneously with both WT DHFR and antifolate resistant DHFR mutants. Importantly, the development of a biophysics-based quantitative model for the fitness landscape of DHFR (Rodrigues et al., 2016; Rodrigues and Shakhnovich, 2019) is a valuable tool to identify the target resistant mutants that are the best candidates for drug design based on biophysical parameters of mutant proteins. The current work presents a design protocol and a multi-tool drug development pipeline which is motivated by these evolutionary understandings and presents a plausible strategy which aims at inhibiting both WT and evolutionary escape variants.

By an efficient virtual screening protocol to scan large databases, two hits of novel scaffolds were identified, which showed inhibitory activity against both WT and resistant mutant forms of DHFR. Unlike conventional approaches primarily focused on chemical synthesis of derivatives based on known inhibitor scaffolds (Francesconi et al., 2018; Hopper et al., 2019; Lam et al., 2014; Reeve et al., 2016), we deployed a multi-tier approach to come up with chemically novel inhibitors. To further improve DHFR inhibitory activity of the two hits, a rapid round of compound optimization was conducted through a structural similarity search. This approach turned out to be effective as we tested only 12 candidate compounds and found CD15-3 with improved antimicrobial activity against both the WT and trimethoprim-resistant DHFR mutant $E$. coli strains. Among trimethoprim-resistant mutations, P21L, A26T, L28R, and their combinations recurrently appeared in two out of five independent evolution experiments, and their order of fixation in both cases was similar (Toprak et al., 2012). Fitness landscape of TMP resistance showed that the L28R variant is most resistant among all three single mutants (Rodrigues et al., 2016). Introduction of another mutation on the L28R background, either P21L or A26T, does not change IC50 significantly (Rodrigues et al., 2016; Toprak et al., 2012). Thermal denaturation experiments demonstrate that L28R mutation is stabilizing showing an increase in Tm of $6{ }^{\circ} \mathrm{C}$ above WT DHFR, in contrast to the destabilizing mutations P21L and A26T (Rodrigues et al., 2016). In addition, the L28R mutation cancels out the destabilization brought by P21L and A26T, restoring the Tm of the double and triple mutants to WT values. The L28R mutation also shows improved compactness, inferred from its reduced bis-ANS binding (Rodrigues et al., 2016). Thus, among the evolved mutant E. coli strains, L28R is one of the most frequently encountered resistance mutations in folA gene which render traditional anti-folate drugs like TMP clinically ineffective (Manna et al., 2021; Rodrigues et al., 2016; Toprak et al., 2012). Effectively L28R serves as a stability "reservoir" providing a gateway to multiple evolutionary trajectories leading to resistance. The discovered DHFR inhibitor CD15-3 shows about 3-fold better efficacy than that of TMP on L28R E. coli variant strain, indicating its potential in combating resistance conferred by gateway point mutations of DHFR.

Our in vivo DHFR over-expression experiment showed partial recovery from the CD15-3 induced growth inhibition, thereby validating DHFR as a target for the intracellular inhibition by 
CD15-3. That DHFR is being targeted by CD15-3 is further supported by our imaging results showing antibiotic-stress induced filamentation in the WT cells, a clear sign indicating that the folate pathway is indeed impacted by the drug(Bhattacharyya et al., 2021). However, the partial recovery upon DHFR overexpression strongly suggests the presence of at least one additional protein target of CD15-3. As CD15-3 was designed to allow interaction with both WT and "modified" active site pockets in the mutant forms of DHFR, this might make CD15-3 active against other enzymes that bind structurally similar substrates, most likely of the folate pathway. In a follow up work we present integrative metabolomic analysis that allowed us to identify additional targets of CD15-3 (Chowdhury et al., 2021) (

In order to assess the validity of the strategy do design compounds that block escape routes we carried out 30-day long evolution experiments to evolve E. coli under CD15-3 unduced stress. Unlike TMP resistance which emerged quickly and resulted in strains having 8 to 200 -fold higher IC50, under CD15-3 E. coli evolved IC50 which was only 2.7 times greater than WT. Further our whole genome sequence analysis revealed that there was no mutation in the target folA gene. Rather, we found that partial genome duplication of the region containing efflux pumps was responsible for evolution of modest resistance against CD15-3. The importance of efflux pump genes and transporters as first line of defence against antibiotic stressors was shown in studies where deletion of such genes leads to inferior mutational paths and escape strategies (Lukačišinová et al., 2020). The generic resistance phenotype observed in CD15-3 evolved cells could be potentially attributed to the multivalent nature of CD15-3. TMP specifically interacts with DHFR and hence mutation in the target loci (folA) provides an optimal solution to escape the TMP stress. As CD15-3 potentially interacts with at least one more target besides DHFR, point mutations in one of the target proteins do not provide optimal solutions. While point mutations at multiple target loci can complicate the evolutionary fitness landscape, efflux mediated resistance on the other hand provides a generic and rapid rescue strategy bypassing the need of multiple point mutations. However, this strategy apparently has its drawback as overexpression of additional pumps can incur fitness cost and lead to only modest 2-3 fold increase in $\mathrm{IC}_{50}$.

Bacterial fitness/adaptation landscape under conditions of antibiotic induced stress could be complicated with multiple escape strategies. The method and the plausible solutions presented here aim to block the common antifolate evolutionary escape route through missense mutations in the folA locus. Indeed CD15-3 was effective in blocking the escape routes involving target modifications by inhibiting the most potent DHFR escape mutants along with the WT form. Surprisingly, CD15-3 was equally effective in inhibiting growth of the TMP escape mutants which were not explicitly modeled at the computational design stage of this project such as D27E. CD15-3 also showed modest inhibition of W30R and W30C mutants emerged under TMP selection with $\mathrm{IC}_{50}$ values less than two-fold higher than the WT. It is interesting to note that the CD15-3 inhibitory activity extends beyond the DHFR variants that were initially selected as targets for structure-based design and that "unplanned" D27E and W30 mutants get inhibited with WT like efficacy. 
Exploration of long-term evolutionary mechanism of potential escape from CD15-3 can help us understand the complexity of fitness landscape for this novel prospective antibiotic. This can further help in refining the drug development protocol which can also address the issue of efflux mediated evolutionary escape routes. A plausible futuristic anti-evolution strategy could be using combination of potential evolution drug formulations like CD15-3 and adjuvants which could block the pumps, thereby potentially also obstructing the generic escape routes. Further this antievolution strategy in principle would be interesting to explore in the context of the currently known combination-drug therapy protocols extending its scope relevance and application. As CD15-3 has additional non-DHFR cellular target, it could serve as a monotherapy-mimic of a combination drug cocktail whose design is guided by evolutionary understanding based on recent insights into a short term bacterial adaptation. We suggest that the currently popular drug development protocols could be improved to proactively account, at least partly, for bacterial adaptation.

As a caveat we note that novel compounds discovered here, while promising as candidates for new class of anti-evolution antibiotics, represents only initial leads in the drug development pipeline. A detailed characterization of pharmacokinetics of the prospective compounds using the standard approaches adopted in pharmaceutical industry needs to be carried out to establish their plausibility as drug candidates. However, our approach that integrates computational and in vitro experimental components in a mutual feedback loop allows considerable power and flexibility in optimization of emerging compounds potentially significantly shortening the development cycle.

To summarize, we believe that the presented comprehensive multiscale-multitool approach can be an attractive starting point for further optimization and development of evolution drugs and adopting a broad strategy to combat evolution of drug resistance.

\section{Methods and Materials}

\section{Preparation of compound databases}

Two commercially available databases ChemDiv (http://www.chemdiv.com/, about 1.32 million compounds) and Life Chemicals (http://www.lifechemicals.com/, about 0.49 million compounds) were selected for virtual screening. Both databases contain a large amount of diverse structures which are useful as potential hits for drug development and providing integrative drug discovery strategies for pharmaceutical and biotech companies (Zhang et al., 2015). In addition, the known E.coli. DHFR inhibitors (183 compounds) with $\mathrm{IC}_{50}$ values ranging from $1 \mathrm{nM}$ to $100 \mu \mathrm{M}$ were downloaded from BindingDB (https://www.bindingdb.org/bind/index.jsp)(Liu et al., 2006) used as a reference database. All compounds from the two databases were added hydrogens and then optimized by a user-defined protocol in Pipeline Pilot (Warr, 2012). A couple of physicochemical properties including molecular weight, AlogP, number of hydrogen donors (HBD) and acceptors (HBA), number of rings, aromatic rings, rotatable bonds and molecular fractional polar surface area (Molecular_FPSA) were calculated in Pipeline Pilot (Warr, 2012) using the Calculate Molecular Properties protocol. All the compounds were further prepared by 
the LigPrep module in Maestro 10.2 in Schrodinger (Steffan and Kuhlen, 2001) to ensure they were appropriate for molecular docking.

\section{Molecular Docking}

All E. coli DHFR crystal structures (issued before 09-10-2016) were downloaded from Protein Data Bank (PDB)(Berman et al., 2006) and classified into three categories. Among them four representatives including PDB 1RX3 (M20 loop closed), 1RA3 (M20 loop open), 1RC4 and 5CCC (M20 loop occluded) were selected for molecular docking and molecular dynamics studies. All these protein structures were prepared by the Protein reparation wizard in Maestro 10.2 of Schrodinger (Madhavi Sastry et al., 2013) to add hydrogens and missing atoms and to minimize the energy. All water molecules in the original crystal structures were removed except the $302^{\text {th }} \mathrm{H}_{2} \mathrm{O}$ which is located in the active site and forms a key hydrogen bond with the cognate ligand. Hydrogen atoms and partial charges were added to the protein, and then a restrained minimization of only the hydrogens was conducted. A grid box centered on the cognate ligand was generated by the Receptor Grid Generation module in Schrodinger. To soften the potential for nonpolar parts of the receptors, one can scale the van der Waals radii (scaling factor set to 1.0) of the receptors with partial atomic charge less than the specified cutoff (set to $0.25 \AA$ ). Due to its excellent performance through a self-docking analysis (Zhang et al., 2017; Zhang et al.), the Glide module in Schrodinger was selected for molecular simulations. All three precision modes including the high throughput virtual screening (HTVS), the standard precision (SP) and the extra precision (XP) were utilized sequentially according to their speed and accuracy. For each docking run, the 10 best poses of each ligand were minimized by a post docking program with the best pose saved for further analysis and the root mean standard deviation (RMSD) between the output and input structures were calculated.

\section{Molecular Dynamics}

Protein-compound complexes resulted from molecular docking were employed for molecular dynamics simulation. Proteins were prepared using the Protein Preparation Wizard (Madhavi Sastry et al., 2013) tool of Maestro in Schrodinger by adding missing atoms and hydrogens and minimizing the energy. Ligands were preprocessed by assigning the correct protonation state using Chimera 1.9 (Pettersen et al., 2004). Then, they were optimized by an implicit water model under the M06-2X/6-31+G** level of theory and their RESP charges were computed at the HF/6-31G* level of theory using Gaussian 09 (Chai and Head-Gordon, 2009). Finally, their ligand topologies were generated by acpype which is an interface to antechamber(Wang et al., 2006). The TIP3P water model (Jorgensen, 1981) and AMBER03 force field (Chillemi et al., 2010) were used for the simulation using GPU processors with GROMACS, version 5.0.2 (Spoel et al., 2005). Firstly, the system was solvated in a water filled rhombic dodecahedron box with at least $12 \AA$ distance between the complex and box edges. Then, the charges of system were neutralized by adding counter ions $(\mathrm{Na}+$ or $\mathrm{Cl}-)$ by the genion tool in Gromacs. The system was then relaxed through an energy minimization process with steepest descent algorithm first, followed by a conjugate gradient algorithm. The energy of the system was minimized until the maximum force reached $5.0 \mathrm{~kJ} \mathrm{~mol}^{-1} \mathrm{~nm}^{-1}$. After a primary constraint NVT simulation of $200 \mathrm{ps}$ with protein atoms being fixed at $100 \mathrm{~K}$, an NVT simulation of 400 ps without restraints was 
performed with simulated annealing at the temperature going from 100 to $300 \mathrm{~K}$ linearly. Then we performed an NPT simulation of 500 ps to equilibrate the pressure. Eventually, a production MD was conducted for $10 \mathrm{~ns}$ at $300 \mathrm{~K}$ twice. Bond lengths were constrained using LINCS algorithm during the simulation (Hess, 2008). It is commonly accepted that nanosecond MD simulations are reliable to investigate local conformational changes (Yeggoni et al., 2014). Thus, the last $2 \mathrm{~ns}$ of each $10 \mathrm{~ns}$ production simulation were extracted at every 10 ps interval (400 snapshots in total) for calculating the molecular mechanics/Poisson-Boltzmann surface area binding energy (MM-PBSA) by the AMBER12 package(Miller et al., 2012). Average energy for 400 snapshots was saved for binding free energy calculation using MMPBSA (Cheron and Shakhnovich, 2017). More details about this protocol are described elsewhere (Cheron and Shakhnovich, 2017).

\section{Crystal structure selection}

Through visual inspection of M20 loop conformation for the 61 crystal structures of DHFR (before 2016-9-26), crystal structures of three categories of M20 loops were obtained. From Figure S1, a total of 38 closed, 11 open, 12 occluded crystal structures of E.coli DHFR as well as the comparison of them are shown. The NADPH/NADP+ as well as the substrates (most of them are MTX) are also shown. It can be seen that M20 loop constitutes part of the substrate binding site, so it will influence the binding of the substrates and its inhibitors. It has shown that the closed conformation is adopted when the substrate and cofactor are both bound (Agarwal et al., 2002), where the M20 loop is packed against the nicotinamide ring of the cofactor. This is the only conformation where substrate and cofactor are arranged favorably for reaction and the following state of the catalytic cycle (Agarwal et al., 2002) and also the only conformation that can be found in DHFR from all other species, regardless of the crystal packing and ligands bound positions in the binding site (Sawaya and Kraut, 1997). Observed in the product complexes, the occluded M20 loop is an unproductive conformation in which the central part of the M20 loop forms a helix that blocks access to the binding site for the nicotinamide moiety of the cofactor. Thus, the nicotinamide of the cofactor is forced out into solvent, making it unresolved in the crystal structures (Sawaya and Kraut, 1997). The open loop is a conformational intermediate between the extremes of the closed and occluded loops. The M20 loop dynamics plays a significant role in ligand binding and catalysis. It has become an area of great interest due to the persuasive evidence of conformational change in the loop during the catalytic cycle and its interaction with the substrate and cofactor (Sawaya and Kraut, 1997). However, it is still unknown which M20 loop conformation is a binding mode for inhibitors of E. coli DHFR. Whether binding single conformation or multiple conformations of the M20 loop leads to stronger binding is also not known. Thus, it is important to determine the target crystal structures that is most predictive for virtual screening investigation. Considering the conformation M20 loop, crystal structure resolution, as well as completeness of cognate ligands such as MTX or DDF and NADPH, we selected four M20 loop conformations (Closed: 1RX3, Open: 1RA3, Occluded: 1RC4 and 5CCC, Figure S1) for the docking verification. Two occluded PDBs were selected because their M20 loop conformations did not overlap well. The feasibility of each M20 loop conformation was assessed by docking the known 183 E. coli DHFR inhibitors with IC 50 values ranging from $1 \mathrm{nM}$ to $100 \mu \mathrm{M}$ to the four representative PDB structures. As shown in 
Figure S2 and Table 4, for docking score less than -10, the cumulative numbers of selected inhibitors were 22 for the closed M20 1RX3 - greater than for the open M20 1RA3 (10), and occluded M20 1RC4 (1) and 5CCC (13). In particular, when the cutoff of docking score was set to -9 , using closed M20 1RX3 as target resulted in correct prediction of as many as 62 inhibitors compared to the open M20 1RA3 (25), and occluded M20 1RC4 (4) and 5CCC (32). At the higher cutoffs, open M20 1RA3 and occluded M20 5CCC showed comparable trends with that of closed M20 1RX3. However, even the M20 loop of 1RC4 and 5CCC both belong to the occluded conformation, their performance in docking of known ligands was strikingly different. It appears that $1 \mathrm{RC} 4$ performed much worse than $5 \mathrm{CCC}$ and it performed the worst compared to other target conformations. A possible reason could be that M20 loop in 1RC4 structure is closer to the substrate, making the binding pocket smaller to accommodate relatively large inhibitors. Further, we focused on the inhibitors with $\mathrm{IC}_{50}$ values less than $1 \mu \mathrm{M}$. As seen from Figure $\mathrm{S} 2$ and Figure S3, using the closed M20 1RX3 predicted about 58\% (42) out of the total of 72 the inhibitors, while the open M20 1RA3 only predicted 18\% (13), the occluded 1RC4 got 3\% (2) and the occluded 5CCC predicted about 18\% (13). Altogether these results demonstrate that the closed M20 1RX3 is more representative as a target for molecular docking of E.coli DHFR inhibitors.

Table 4 Number of known inhibitors binding DHFR with different M20 loops through molecular docking

\begin{tabular}{|c|c|c|c|c|c|c|c|c|}
\hline \multirow{2}{*}{$\begin{array}{l}\text { XP Docking } \\
\text { Score } \\
\text { Threshold }\end{array}$} & \multicolumn{4}{|c|}{ All inhibitors $\left(\mathrm{IC}_{50}: 1 \mathrm{nM} \sim 240 \mu \mathrm{M}\right)$} & \multicolumn{4}{|c|}{ Inhibitors $\left(\mathrm{IC}_{50}<1 \mu \mathrm{M}\right)$} \\
\hline & $\begin{array}{l}\text { Closed } \\
\text { (1RX3) }\end{array}$ & $\begin{array}{l}\text { Open } \\
\text { (1RA3) }\end{array}$ & $\begin{array}{l}\text { Occluded } \\
\text { (1RC4) }\end{array}$ & $\begin{array}{l}\text { Occluded } \\
\text { (5CCC) }\end{array}$ & $\begin{array}{l}\text { Closed } \\
\text { (1RX3) }\end{array}$ & $\begin{array}{l}\text { Open } \\
\text { (1RA3) }\end{array}$ & $\begin{array}{l}\text { Occluded } \\
\text { (1RC4) }\end{array}$ & $\begin{array}{l}\text { Occluded } \\
\text { (5CCC) }\end{array}$ \\
\hline$<-10$ & 22 & 10 & 1 & 13 & 14 & 4 & 0 & 4 \\
\hline$<-9$ & 62 & 25 & 4 & 32 & 42 & 13 & 2 & 13 \\
\hline$<-8$ & 77 & 77 & 12 & 73 & 48 & 50 & 4 & 35 \\
\hline$<-7$ & 107 & 93 & 21 & 114 & 51 & 53 & 8 & 51 \\
\hline$<-6$ & 148 & 136 & 53 & 167 & 58 & 58 & 22 & 68 \\
\hline$<-5$ & 174 & 168 & 148 & 181 & 69 & 67 & 53 & 72 \\
\hline$<-4$ & 181 & 182 & 182 & 183 & 72 & 72 & 71 & 72 \\
\hline$<0$ & 183 & 183 & 183 & 183 & 72 & 72 & 72 & 72 \\
\hline
\end{tabular}

\section{Construction of binding affinity prediction model}

Earlier, the MD simulation of protein-ligand complexes followed by MM/PBSA assessment of binding affinity were applied in our group to the BACE protease (Cheron and Shakhnovich, 2017). Here, we use a similar protocol for E. coli DHFR. Eight known E. coli DHFR inhibitors (Figure S4) including MTX and TMP as well as other six compounds from Carroll et al (Carroll et al., 2012) with known Kd values were chosen for the construction of binding free energy prediction. The Pearson correlation coefficient $(\mathrm{R})$ between the predicted and experimental binding free energies $(\Delta \mathrm{G}=\mathrm{RT} \ln (\mathrm{Kd})$, where $\mathrm{R}$ is the gas constant and $\mathrm{T}=293.15 \mathrm{~K})$ was used to evaluate the accuracy of the protocol developed in (Cheron and Shakhnovich, 2017). TMP and Cmpd 2 as well as Cmpd 6 (Figure S4) do not have crystal structures, their complexes were from molecular docking (TMP was docked to PDB 1RX3 and Cmpd 2 and 6 were docked to 3QYL). 
We used the available crystal structures of TMP complexed with Staphylococcus aureus DHFR (PDB: 2W9G, sequence identity with E. coli DHFR: 55/162 (34.0\%) and sequence similarity: $92 / 162(56.8 \%))$ as a reference and found that the conformation of TMP docked with E. coli DHFR is only about $0.4 \AA$ different from crystal structure with $S$. aureus DHFR (Figure S5), suggesting the accuracy of docked conformation for TMP. We first conducted 2 replicates of 10 ns simulation and then extended simulation length to 20 replicates. Two replicates of $10 \mathrm{~ns}$ simulation provided a quite strong correlation $(\mathrm{R}=0.942)$ between the computational and experimental binding free energies (Figure 1B) essentially indistinguishable from longer simulation comprised of 20 replicates of 10 ns runs in Figure S6). Thus, in subsequent simulations a protocol consisting of two replicates of $10 \mathrm{~ns}$ was adopted. It can also be observed that the binding free energies followed a normal distribution (Figure S6) in both the short and long simulations, which indicates that the use of mean value to represent the general binding free energy is reasonable. An evolutionary study (Oz et al., 2014; Toprak et al., 2012) of TMP resistance found that three key resistance mutations P21L, A26T, L28R, and their combinations constitute a set that recurrently occurred in two out of five independent evolution experiments, and their order of fixation in both cases was similar. Although mechanisms leading to TMP resistance could be extremely complex and diverse, these sets of point mutations represent a simplistic model of in vitro evolution. These point mutations are located within the binding pocket of dihydrofolate substrate within a small region of eight residues in the DHFR protein that constitutes a flexible Met-20 loop (residues 9-24) and an $\alpha$-helix (residues 25-35). Thus, the correlation coefficients between the computational and experimental binding free energies for TMP for the three mutants as well as their double and triple combinations were calculated. In addition, linear regression equation models to predict binding free energy for TMP against mutant DHFR originated from Listeria grayi and Chlamydia muridarum were also included. As shown in Figure S7, from two replicates of $10 \mathrm{~ns}$ simulation, the correlation coefficient (R) for $L$. grayi and C. muridarum DHFR were 0.895 and 0.839 , respectively. The reason why the prediction for L. grayi and C. muridarum is somewhat inferior to E. coli $\mathrm{DHFR}(\mathrm{R}=0.953$ from Figure $1 \mathrm{C}$ in the manuscript) may be that the protein-ligand complexes used for MD simulation are derived from molecular modeling rather than from crystal structures. Still, the values of binding free energy follow a normal distribution. Those models were used for scoring of virtual screening hits later.

\section{Selection of virtual screening hits}

Structure-based virtual screening (SBVS) can quickly select compounds with reasonable binding patterns and higher predicted scores from a large number of compounds. According to the workflow (Figure 1A in the manuscript), a total of about 1.8 million compounds from ChemDiv and Life Chemicals were screened for compliance with the Lipinski's rule of five (Manto et al., 2018), resulting in about 1.5 million compounds. Then, a virtual screening process with three steps of different speed and precision including high throughput virtual screening (HTVS), standard precision (SP) and extra precision (XP) were respectively applied to the 1.5 million compounds. By setting different cutoff values for the HTVS of -5, SP of -7 and XP of -5, about 2900 compounds were obtained. The protein-ligand interaction of the crystal structures showed that Asp27 within the binding pocket is one of the most critical for forming hydrogen bond with 
known inhibitors. A total of 491 hits having contacts with Asp27 were filtered. After visual selection, 307 out of 491 compounds were submitted for the molecular dynamics evaluation using the previously selected DHFR crystal structure PDB 1RX3 as a target. Using the protocol described above we predicted the binding affinities of all 307 compounds. With a cutoff value of less than $-20 \mathrm{kcal} / \mathrm{mol}, 40$ compounds were chosen for further analysis. For those 40 compounds with 32 from the ChemDiv database and 8 from the Life Chemicals database we calculated their binding affinity for the WT and three single, three double and one triple mutants based on P21L, A26T and L28R. Calculations predicted significant binding affinity for all compounds not only to the wild type DHFR, but also to the DHFR mutants.

Based on the principle that compounds with similar properties tend to have similar activity (Kumar, 2011), and to ensure that the selected compounds with chemical novelty, three types of compound similarity based on Tanimoto coefficient (Zhang et al., 2013) were compared between the selected hits with that of the known 183 DHFR inhibitors. The Tanimoto coefficient uses the ratio of the intersecting set to the union set as the measure of similarity when each attribute is expressed in binary. Represented as a mathematical equation:

$$
\mathrm{T}_{\mathrm{AB}}=\frac{\mathrm{c}}{a+b-c}
$$

In this equation, individual fingerprint bits set in molecules $A$ and $B$ are represented by a and $\mathrm{b}$, respectively; and $\mathrm{c}$ is the intersection set. $\mathrm{T}_{A B}$ value ranges from 0 to 1 , where 0 represents that no same bits are detected; however, 1 does not mean that the two molecules are totally identical. Two-dimensional (2D) physicochemical properties including molecular weight, AlogP, polar surface area, number of rotatable bonds, rings, aromatic rings, hydrogen acceptors and hydrogen donors were compared from the two-dimensional property's aspect. Interaction similarity based on protein-ligand interaction (Huovinen et al., 1995) were calculated to ensure that the selected compounds form similar interactions with the critical binding site pocket residues which can further guarantee their biological activity. ECFP4 (Zhang et al., 2013), an extended connectivity fingerprints which can represent the chemical diversity of compounds, was used to ensure the wide chemical space and novelty in their structures. As shown in Figure S8, the 2D physicochemical properties similarity were concentrated in the range of 0.6 to 0.8 and the protein-ligand interaction fingerprint (PLIF) similarities were mainly distributed in the range of 0.7 to 0.9 on the one hand, and the ECFP4 chemical similarities were only just between 0.2 to 0.3 on the other hand. This indicated that the selected hits were of similar 2D physicochemical property and protein-ligand binding interaction but also possessed chemical diversity compared with the known DHFR inhibitors. For detailed analysis of the 2D properties for the selected hits, the predicted values above mentioned properties including molecular weight, AlogP, polar surface area, number of rotatable bonds, rings, aromatic rings, hydrogen acceptors and hydrogen donors were compared with that of the known DHFR inhibitors. It can be clearly seen from Figure S9 that those 2D properties showed similar distributions with the positive controls, further proving the effectiveness of the selected hits. Finally, selected 40 hit compounds were submitted for purchase and biological activity evaluation. 
All reagents and chemicals were of high quality and were procured from Sigma-Aldrich Co., USA, Amresco, or Fisher Scientific. According to our former study, wild-type (WT) E.coli, C. muridarum and L. grayi DHFR as well as mutant E. coli DHFR proteins including P21L, A26T and L28R point mutants were expressed and purified in the same way as previously described (Rodrigues et al.; Rodrigues et al., 2016). Human DHFR was purchased from Sigma Aldrich. In an initial screen, the compounds were assayed for inhibition of DHFR catalytic activity at a single fixed concentration of $200 \mu \mathrm{M}$. The reaction mixtures $(100 \mu \mathrm{L}$ final volume) contained $100 \mu \mathrm{M}$ NADPH, $30 \mu \mathrm{M}$ dihydrofolate and $100 \mathrm{mM}$ HEPES, $\mathrm{pH}$ 7.3. The DHFR enzyme was added to initiate the reaction $(16.7 \mathrm{nM}$ final concentration) and the oxidation of NADPH was monitored at $25^{\circ} \mathrm{C}$ by the decrease in absorbance at $340 \mathrm{~nm}$. The initial velocities with product formation less than 5\% were measured and the nonenzymatic hydrolysis of NADPH was subtracted. The concentration-dependent inhibition curves were determined at variable concentration of each inhibitor. To minimize the interference at high compound concentrations, the rate of NADPH oxidation catalysed by DHFR was instead monitored by following the decrease in fluorescence at $450 \mathrm{~nm}$ (excitation $340 \mathrm{~nm}$ ). Inhibition constants were determined from kinetic competition experiments performed based on varying inhibitor concentrations (Srinivasan and Skolnick, 2015). The curves were fit to the equation $y=\frac{100 \%}{\left(1+I / I C_{50}\right)}$, where I

is concentration of the inhibitor and $y$ is the percentage of catalytic activity of DHFR. The values of inhibition constant $K_{i}$ were obtained by fitting a competitive inhibition equation (Krohn and Link, 2003) from plots of activity vs. inhibitor concentration using the equation $K_{i}=\frac{I C_{50}}{1+\frac{[S]}{K_{M}}}$, where the $K_{M}$ values of DHFR enzyme for both substrates (DHF and NADPH) have been measured before (Rodrigues et al., 2016) and [S] is the substrate concentration of DHF. All the measurements were conducted in triplicate, and the error values are indicated by standard errors. All the data were fit using the nonlinear curve-fitting subroutines OriginPro (Seifert, 2014)

\section{Bacterial Growth Measurements and Determination of IC50 values}

Cultures in M9 minimal medium was grown overnight at $37{ }^{\circ} \mathrm{C}$ and were then normalized to an $\mathrm{OD}$ of 0.1 using fresh medium. A new normalization to an $\mathrm{OD}=0.1$ was conducted after additional growth during 5-6 h. Then, the M9 medium and six different concentrations of the positive control TMP and all hit compounds in the 96 -well plates (1/5 dilution) were incubated. The incubation of the plates was performed at $37{ }^{\circ} \mathrm{C}$ and the orbital shacking and absorbance measurements at $600 \mathrm{~nm}$ were taken every 30 min during $15 \mathrm{~h}$. By integration of the area under the growth curve (OD vs. time), the growth was quantified between 0 and $15 \mathrm{~h}$, as described elsewhere (Palmer et al., 2015a). For the WT DHFR or a given mutant, the growth integrals normalized by corresponding values of growth for that same strain without the hit compounds. By fitting a logistic equation to plots of growth vs. compound concentrations, the $\mathrm{IC}_{50}$ values 
were determined. The reported $\mathrm{IC}_{50}$ values are an average based on at least three replicates and standard errors are indicated.

\section{Whole Genome sequencing}

Using single isolated colonies, whole genome sequencing for the evolved variants was performed resorting to Illumina MiSeq 2 × 150 bp paired-end configuration (Novogene). We used breqseq pipeline (Deatherage et al., 2014) on default settings, using the E. coli K-12 substr. BW25113 reference genome (GenBank accession no. CP009273.1).

\section{Evolution experiments}

Evolution of E. coli strains in the presence of prospective compound CD15-3 or TMP was carried out by serial passaging using an automated liquid handling system (Tecan Freedom Evo 150), a procedure similar to the one described previously (Rodrigues and Shakhnovich, 2019). In this setup, cultures are grown in wells of a 96-well microplate, and the optical density (600 nm) is measured periodically using a plate reader (Tecan Infinite M200 Pro). In each serial passage, the cultures are diluted with fresh growth media into the wells of the adjacent column. The growth rate measured for each culture at a given passage is compared with the growth rate determined in the absence of antibiotic and the concentration of antibiotic is increased by a factor of 2 if it exceeds $(75 \%)$, otherwise maintained. To avoid excessive antibiotic inhibition, the concentration was increased only once in every two consecutive passages. This procedure forces cells to grow under sustained selective pressure at growth rates close to $50 \%$ of that of noninhibited cells.

For plate assays WT cells were grown for around 8 hours without any antibiotic selection. Cells were grown in M9 media supplemented with $0.8 \mathrm{GL}^{-1}$ glucose. The cells were thereafter plated on M9 agar plates with similar glucose and M9 salt concentraions as was in the M9 liquid media. Plating was done after 1000 fold dilution of the growing culture. The plates were having either TMP or CD15-3 as the selection inducer and their respective concentrations were $3 \mathrm{X}$ of $\mathrm{IC}_{50}$ values as is known for WT/naïve cells (viz. $1.2 \mu \mathrm{g} / \mu 1$ for TMP and $210 \mu \mathrm{M}$ for CD15-3). It is to be noted that 6 well plates were used for this study and the plated were incubated at $37^{0} \mathrm{C}$ for colonies to appear.

\section{Differential interference contrast (DIC)}

WT cells were grown in M9 media supplemented with $0.8 \mathrm{gL}^{-1}$ glucose and casamino acids (mixtures of all amino acids except tryptophan) in absence and presence of CD15-3 at $42{ }^{\circ} \mathrm{C}$ for incubation and $300 \mathrm{rpm}$ constant shaking. Drop in DHFR activity has been associated with cellular filamentation and similar phenotype is observed under TMP treatment(Bhattacharyya et al., 2019). Since CD15-3 targets intracellular DHFR and soluble fraction of cellular DHFR is lower at 42 degrees $\mathrm{C}$ we chose this temperature for our imaging studies (Bershtein et al., 2012).

Aliquots were taken from the growing culture and they were drop casted on agar bed/blocks. These blocks were taken further processed for differential inference contrast (DIC) imaging using Zeis Discovery imaging workstation. Multiple fields were observed and scanned for a single condition type and a minimum of three replicates were used for imaging studies. Similar 
methods for imaging were used for evolved cell types under conditions of absence and presence of CD15-3 compound. Intellesis Module was used for analyzing DIC images. On average, around 500 cells were analyzed for computing cell length. E. coli cell lengths in our imaging studies were not normally distributed. Nonparametric Mann-Whitney test was therefore used to determine if the cell length distributions were significantly different upon CD15-3 treatment.

Acknowledgement This work was supported by NIH RO1 068670 and by the program of China Scholarship Council (CSC). We are grateful to Nicolas Chéron and Jiho Park for help with the computational setup.

\section{References}

Agarwal, P.K., Billeter, S.R., Rajagopalan, P.T., Benkovic, S.J., and Hammes-Schiffer, S. (2002). Network of coupled promoting motions in enzyme catalysis. Proc Natl Acad Sci U S A 99, 2794-2799.

Ahmad, S., Kirk, S., and Eisenstark, A. (1998). Thymine metabolism and thymineless death in prokaryotes and eukaryotes. Annual review of microbiology 52, 591-625.

Baell, J.B., and Holloway, G.A. (2010). New substructure filters for removal of pan assay interference compounds (PAINS) from screening libraries and for their exclusion in bioassays. Journal of medicinal chemistry 53, 2719-2740.

Baym, M., Stone, L.K., and Kishony, R. (2016). Multidrug evolutionary strategies to reverse antibiotic resistance. Science 351.

Berman, H.M., Westbrook, J., Feng, Z., Gillil, G., Bhat, T.N., Weissig, H., Shindyalov, I.N., and Bourne, P.E. (2006). The Protein Data Bank, 1999\&ndash.

Bershtein, S., Mu, W., and Shakhnovich, E.I. (2012). Soluble oligomerization provides a beneficial fitness effect on destabilizing mutations. Proceedings of the National Academy of Sciences 109, 4857-4862.

Bharath, Srinivasan, João, V., Rodrigues, Sam, Tonddast-Navaei, Eugene, Shakhnovich, and Jeffrey (2017). Rational Design of Novel Allosteric Dihydrofolate Reductase Inhibitors Showing Antibacterial Effects on Drug-Resistant Escherichia coli Escape Variants. Acs Chem Biol 1848-1857, 12.

Bhattacharyya, S., Bershtein, S., Adkar, B.V., Woodard, J., and Shakhnovich, E.I. (2019). A case of 'mistaken identity': structurally similar ligand inhibits Thymidylate Kinase causing reversible filamentation of $<$ em $>$ E. coli</em>. bioRxiv, 738823.

Bhattacharyya, S., Bershtein, S., Adkar, B.V., Woodard, J., and Shakhnovich, E.I. (2021). Metabolic response to point mutations reveals principles of modulation of in vivo enzyme activity and phenotype. Molecular systems biology in press, 738823.

Bhattacharyya, S., Bershtein, S., Yan, J., Argun, T., Gilson, A.I., Trauger, S.A., and Shakhnovich, E.I. (2016). Transient protein-protein interactions perturb E. coli metabolome and cause gene dosage toxicity. Elife 5, e20309.

Bleyer, W.A. (2015). The clinical pharmacology of methotrexate: new applications of an old drug. Cancer 41, 36-51.

Cao, S., Huseby, D.L., Brandis, G., and Hughes, D. (2017). Alternative evolutionary pathways for drugresistant small colony variant mutants in Staphylococcus aureus. MBio 8.

Carroll, M.J., Mauldin, R.V., Gromova, A.V., Singleton, S.F., Collins, E.J., and Lee, A.L. (2012). Evidence for dynamics in proteins as a mechanism for ligand dissociation. Nature chemical biology 8, 246-252.

Chai, J.D., and Head-Gordon, M. (2009). Long-range corrected double-hybrid density functionals. Journal of Chemical Physics 131, 174105. 
Chakraborty, A.K., and Barton, J.P. (2017). Rational design of vaccine targets and strategies for HIV: a crossroad of statistical physics, biology, and medicine. Rep Prog Phys 80, 032601.

Cheron, N., and Shakhnovich, E.I. (2017). Effect of sampling on BACE-1 ligands binding free energy predictions via MM-PBSA calculations. J Comput Chem 38, 1941-1951.

Chillemi, G., Coletta, A., Mancini, G., Sanna, N., and Desideri, A. (2010). An amber compatible molecular mechanics force field for the anticancer drug topotecan. Theoretical Chemistry Accounts 127, 293-302. Chowdhury, S., Zielinski, D.C., Dalldorf, C., Rodrigues, J.V., Palsson, B., and Shakhnovich, E. (2021). A systems-guided approach to discover the intracellular target of a novel evolution-drug lead. bioRxiv, 2021.2005.2017.444532.

Coutinho, H.D., Costa, J.G., Lima, E.O., Falcao-Silva, V.S., and Siqueira-Júnior, J.P. (2010). Increasing of the aminoglicosyde antibiotic activity against a multidrug-resistant $\mathrm{E}$. coli by Turnera ulmifolia $\mathrm{L}$. and chlorpromazine. Biological Research for Nursing 11, 332-335.

Delmar, J.A., Su, C.-C., and Edward, W.Y. (2013). Structural mechanisms of heavy-metal extrusion by the Cus efflux system. Biometals 26, 593-607.

Doud, M.B., Lee, J.M., and Bloom, J.D. (2018). How single mutations affect viral escape from broad and narrow antibodies to $\mathrm{H} 1$ influenza hemagglutinin. Nature communications 9, 1386.

Dzidic, S., and Bedeković, V. (2003). Horizontal gene transfer-emerging multidrug resistance in hospital bacteria. Acta Pharmacologica Sinica 24, 519-526.

Falzone, C.J., Wright, P.E., and Benkovic, S.J. Dynamics of a flexible loop in dihydrofolate reductase from Escherichia coli and its implication for catalysis. Biochemistry 33, 439-442.

Finland, M., and Kass, E.H. (1973). Trimethoprim-sulfamethoxazole. Summary and comments on the conference. Journal of Infectious Diseases 128, Suppl:792.

Forge, A., and Schacht, J. (2000). Aminoglycoside antibiotics. Audiology and Neurotology 5, 3-22.

Francesconi, V., Giovannini, L., Santucci, M., Cichero, E., Costi, M.P., Naesens, L., Giordanetto, F., and Tonelli, M. (2018). Synthesis, biological evaluation and molecular modeling of novel azaspiro dihydrotriazines as influenza virus inhibitors targeting the host factor dihydrofolate reductase (DHFR). European journal of medicinal chemistry 155, 229-243.

Gaebler, C., Wang, Z., Lorenzi, J.C.C., Muecksch, F., Finkin, S., Tokuyama, M., Cho, A., Jankovic, M., Schaefer-Babajew, D., Oliveira, T.Y., et al. (2021). Evolution of antibody immunity to SARS-CoV-2. Nature 591, 639-644.

Gong, L.I., Suchard, M.A., and Bloom, J.D. (2013). Stability-mediated epistasis constrains the evolution of an influenza protein. Elife 2, e00631.

Gudipaty, S.A., Larsen, A.S., Rensing, C., and McEvoy, M.M. (2012). Regulation of Cu (I)/Ag (I) efflux genes in Escherichia coli by the sensor kinase CusS. FEMS microbiology letters 330, 30-37.

Hegreness, M., Shoresh, N., Damian, D., Hartl, D., and Kishony, R. (2008). Accelerated evolution of resistance in multidrug environments. Proceedings of the National Academy of Sciences 105, 1397713981.

Hess, B. (2008). P-LINCS: A parallel linear constraint solver for molecular simulation. Journal of Chemical Theory and Computation 4, 116-122.

Hopper, A.T., Brockman, A., Wise, A., Gould, J., Barks, J., Radke, J.B., Sibley, L.D., Zou, Y., and Thomas, S. (2019). Discovery of Selective Toxoplasma gondii Dihydrofolate Reductase Inhibitors for the Treatment of Toxoplasmosis. Journal of Medicinal Chemistry 62, 1562-1576.

Huovinen, P., Sundström, L., Swedberg, G., and Sköld, O. (1995). Minireview. Trimethoprim and sulfonamide resistance. Antimicrobial Agents \& Chemotherapy 39, 279-289.

Izbicka, E., Diaz, A., Streeper, R., Wick, M., Campos, D., Steffen, R., and Saunders, M. (2009). Distinct mechanistic activity profile of pralatrexate in comparison to other antifolates in in vitro and in vivo models of human cancers. Cancer Chemotherapy \& Pharmacology 64, 993-999. 
Jorgensen, W.L. (1981). Transferable Intermolecular Potential Functions for Water, Alcohols, and Ethers. Application to Liquid Water. Jamchemsoc 103.

Justice, S.S., Hunstad, D.A., Cegelski, L., and Hultgren, S.J. (2008). Morphological plasticity as a bacterial survival strategy. Nature Reviews Microbiology 6, 162-168.

Klein, E.Y., Blumenkrantz, D., Serohijos, A., Shakhnovich, E., Choi, J.M., Rodrigues, J.V., Smith, B.D., Lane, A.P., Feldman, A., and Pekosz, A. (2018). Stability of the Influenza Virus Hemagglutinin Protein Correlates with Evolutionary Dynamics. mSphere 3.

Krohn, K.A., and Link, J.M. (2003). Interpreting enzyme and receptor kinetics: keeping it simple, but not too simple. Nuclear Medicine \& Biology 30, 0-826.

Kumar, A. (2011). Investigation of structures similarity of organic substances. Resonance 16, 61-64. Lam, T., Hilgers, M.T., Cunningham, M.L., Kwan, B.P., Nelson, K.J., Brown-Driver, V., Ong, V., Trzoss, M., Hough, G., Shaw, K.J., et al. (2014). Structure-Based Design of New Dihydrofolate Reductase Antibacterial Agents: 7-(Benzimidazol-1-yl)-2,4-diaminoquinazolines. Journal of Medicinal Chemistry 57, 651-668.

Leonardo, F., Ricardo, D.S., Glaucius, O., and Adriano, A. (2015). Molecular Docking and Structure-Based Drug Design Strategies. Molecules 20, 13384-13421.

Lin, J.T., and Bertino, J.R. (1991). Clinical Science Review: Update on Trimetrexate, a Folate Antagonist with Antineoplastic and Antiprotozoal Properties. Cancer Investigation 9, 159-172.

Liu, F.T., Li, N.G., Zhang, Y.M., Xie, W.C., Yang, S.P., Lu, T., and Shi, Z.H. (2020). Recent advance in the development of novel, selective and potent FGFR inhibitors. European journal of medicinal chemistry 186, 111884.

Liu, T., Lin, Y., Xin, W., Jorissen, R.N., and Gilson, M.K. (2006). BindingDB: a web-accessible database of experimentally determined protein-ligand binding affinities. Nucleic Acids Research, suppl_1.

Louie, R.H.Y., Kaczorowski, K.J., Barton, J.P., Chakraborty, A.K., and McKay, M.R. (2018). Fitness landscape of the human immunodeficiency virus envelope protein that is targeted by antibodies. Proc Natl Acad Sci U S A 115, E564-E573.

Lukačišinová, M., Fernando, B., and Bollenbach, T. (2020). Highly parallel lab evolution reveals that epistasis can curb the evolution of antibiotic resistance. Nature communications 11, 1-14.

Madhavi Sastry, G., Adzhigirey, M., Day, T., Annabhimoju, R., and Sherman, W. (2013). Protein and ligand preparation: parameters, protocols, and influence on virtual screening enrichments. Journal of Computer-Aided Molecular Design 27, 221-234.

Manna, M.S., Tamer, Y.T., Gaszek, I., Poulides, N., Ahmed, A., Wang, X., Toprak, F.C.R., Woodard, D.R., Koh, A.Y., Williams, N.S., et al. (2021). A trimethoprim derivative impedes antibiotic resistance evolution. Nature communications 12, 2949.

Manto, C.C., Sara, M., and Laleh, A. (2018). Drug Metabolites and their Effects on the Development of Adverse Reactions: Revisiting Lipinski's Rule of Five. International Journal of Pharmaceutics 549, 133-149. Marchi, J., Lassig, M., Mora, T., and Walczak, A.M. (2019). Multi-Lineage Evolution in Viral Populations Driven by Host Immune Systems. Pathogens 8.

Marcou, G., and Rognan, D. (2007). Optimizing fragment and scaffold docking by use of molecular interaction fingerprints. Journal of Chemical Information \& Modeling 47, 195-207.

Masi, M., Réfregiers, M., Pos, K.M., and Pagès, J.-M. (2017). Mechanisms of envelope permeability and antibiotic influx and efflux in Gram-negative bacteria. Nature microbiology 2, 1-7.

Miller, B.R., McGee, T.D., Swails, J.M., Homeyer, N., Gohlke, H., and Roitberg, A.E. (2012). MMPBSA.py: An Efficient Program for End-State Free Energy Calculations. Journal of Chemical Theory and Computation 8, 3314-3321.

Nesse, R.M., and Stearns, S.C. (2008). The great opportunity: evolutionary applications to medicine and public health. Evolutionary Applications 1, 28-48. 
Ogbunugafor, C.B., Wylie, C.S., Diakite, I., Weinreich, D.M., and Hartl, D.L. (2016). Adaptive landscape by environment interactions dictate evolutionary dynamics in models of drug resistance. PLoS computational biology 12, e1004710.

Oz, T., Guvenek, A., Yildiz, S., Karaboga, E., Tamer, Y.T., Mumcuyan, N., Ozan, V.B., Senturk, G.H., Cokol, M., Yeh, P., et al. (2014). Strength of selection pressure is an important parameter contributing to the complexity of antibiotic resistance evolution. Mol Biol Evol 31, 2387-2401.

Palmer, A.C., Toprak, E., Baym, M., Kim, S., and Kishony, R. (2015a). Delayed commitment to evolutionary fate in antibiotic resistance fitness landscapes. Nature communications 6, 7385.

Palmer, A.C., Toprak, E., Baym, M., Kim, S., Veres, A., Bershtein, S., and Kishony, R. (2015b). Delayed commitment to evolutionary fate in antibiotic resistance fitness landscapes. Nature communications 6 , 1-8.

Peneş, N.O., Muntean, A.A., Moisoiu, A., Muntean, M.M., Chirca, A., Bogdan, M.A., and Popa, M.I. (2017). An overview of resistance profiles ESKAPE pathogens from 2010-2015 in a tertiary respiratory center in Romania. Rom J Morphol Embryol 58, 909-922.

Pettersen, E.F., Goddard, T.D., Huang, C.C., Couch, G.S., Greenblatt, D.M., Meng, E.C., and Ferrin, T.E. (2004). UCSF Chimera-A visualization system for exploratory research and analysis. Journal of Computational Chemistry 25, 1605-1612.

Ragheb, M.N., Thomason, M.K., Hsu, C., Nugent, P., Gage, J., Samadpour, A.N., Kariisa, A., Merrikh, C.N., Miller, S.I., and Sherman, D.R. (2019). Inhibiting the evolution of antibiotic resistance. Molecular cell 73, 157-165. e155.

Reeve, S.M., Scocchera, E., Ferreira, J.J., N, G.D., Keshipeddy, S., Wright, D.L., and Anderson, A.C. (2016). Charged Propargyl-Linked Antifolates Reveal Mechanisms of Antifolate Resistance and Inhibit Trimethoprim-Resistant MRSA Strains Possessing Clinically Relevant Mutations. J Med Chem 59, 64936500.

Rodrigues, J.O.V., Bershtein, S., Li, A., Lozovsky, E.R., Hartl, D.L., and Shakhnovich, E.I. Biophysical principles predict fitness landscapes of drug resistance. Proceedings of the National Academy of Sciences, 201601441.

Rodrigues, J.V., Bershtein, S., Li, A., Lozovsky, E.R., Hartl, D.L., and Shakhnovich, E.I. (2016). Biophysical principles predict fitness landscapes of drug resistance. Proc Natl Acad Sci U S A 113, E1470-1478.

Rodrigues, J.V., and Shakhnovich, E.I. (2019). Adaptation to mutational inactivation of an essential gene converges to an accessible suboptimal fitness peak. Elife 8, e50509.

Roemhild, R., and Schulenburg, H. (2019). Evolutionary ecology meets the antibiotic crisis: Can we control pathogen adaptation through sequential therapy? Evolution, medicine, and public health 2019, 37-45.

Rotem, A., Serohijos, A.W.R., Chang, C.B., Wolfe, J.T., Fischer, A.E., Mehoke, T.S., Zhang, H., Tao, Y., Lloyd Ung, W., Choi, J.M., et al. (2018). Evolution on the Biophysical Fitness Landscape of an RNA Virus. Mol Biol Evol 35, 2390-2400.

Sánchez-Osuna, M., Cortés, P., Llagostera, M., Barbé, J., and Erill, I. (2020). Exploration into the origins and mobilization of di-hydrofolate reductase genes and the emergence of clinical resistance to trimethoprim. Microbial genomics 6 .

Sandegren, L., and Andersson, D.I. (2009). Bacterial gene amplification: implications for the evolution of antibiotic resistance. Nature Reviews Microbiology 7, 578-588.

Sangurdekar, D.P., Zhang, Z., and Khodursky, A.B. (2011). The association of DNA damage response and nucleotide level modulation with the antibacterial mechanism of the anti-folate drug trimethoprim.

BMC genomics 12, 583.

Sawaya, M.R., and Kraut, J. (1997). Loop and subdomain movements in the mechanism of Escherichia coli dihydrofolate reductase: crystallographic evidence. 36, 586. 
Schweitzer, B.I., Dicker, A.P., and Bertino, J.R. (1990). Dihydrofolate reductase as a therapeutic target. Faseb Journal 4, 2441-2452.

Seifert, E. (2014). OriginPro 9.1: Scientific Data Analysis and Graphing Software Software Review (ACS Publications).

Silver, L.L. (2007). Multi-targeting by monotherapeutic antibacterials. Nature Reviews Drug Discovery 6, 41-55.

Singer, S., Ferone, R., Walton, L., and Elwell, L. (1985). Isolation of a dihydrofolate reductase-deficient mutant of Escherichia coli. Journal of bacteriology 164, 470-472.

Spoel, D.V.D., Lindahl, E., Hess, B., Groenhof, G., and Berendsen, H.J.C. (2005). GROMACS: fast, flexible, and free. Journal of Computational Chemistry 26, 1701-1718.

Srinivasan, B., and Skolnick, J. (2015). Insights into the slow - onset tight - binding inhibition of Escherichiacoli dihydrofolate reductase: detailed mechanistic characterization of pyrrolo[3,2 f]quinazoline - 1,3 - diamine and its derivatives as novel tight - binding inhibitors. Febs Journal 282, 1922-1938.

Stearns, S.C. (2012). Evolutionary medicine: its scope, interest and potential. Proceedings of the Royal Society B: Biological Sciences 279, 4305-4321.

Steffan, R., and Kuhlen, T. (2001). MAESTRO: a tool for interactive assembly simulation in virtual environments. Paper presented at: Eurographics Conference on Virtual Environments \& Immersive Projection Technology.

Tamer, Y.T., Gaszek, I.K., Abdizadeh, H., Batur, T.A., Reynolds, K.A., Atilgan, A.R., Atilgan, C., and Toprak, E. (2019). High-Order Epistasis in Catalytic Power of Dihydrofolate Reductase Gives Rise to a Rugged Fitness Landscape in the Presence of Trimethoprim Selection. Molecular Biology and Evolution 36, 15331550.

Tian, J., Woodard, J.C., Whitney, A., and Shakhnovich, E.I. (2015). Thermal stabilization of dihydrofolate reductase using monte carlo unfolding simulations and its functional consequences. PLoS Comput Biol 11, e1004207.

Toprak, E., Veres, A., Michel, J.-B., Chait, R., Hartl, D.L., and Kishony, R. (2012). Evolutionary paths to antibiotic resistance under dynamically sustained drug selection. Nature genetics 44, 101-105.

Toulouse, J.L., Edens, T.J., Alejaldre, L., Manges, A.R., and Pelletier, J.N. (2017). Integron-associated DfrB4, a previously uncharacterized member of the trimethoprim-resistant dihydrofolate reductase $B$ family, is a clinically identified emergent source of antibiotic resistance. Antimicrobial agents and chemotherapy 61.

Tyers, M., and Wright, G.D. (2019). Drug combinations: a strategy to extend the life of antibiotics in the 21st century. Nature Reviews Microbiology 17, 141-155.

Vestergaard, M., Paulander, W., Marvig, R.L., Clasen, J., Jochumsen, N., Molin, S., Jelsbak, L., Ingmer, H., and Folkesson, A. (2016). Antibiotic combination therapy can select for broad-spectrum multidrug resistance in Pseudomonas aeruginosa. International journal of antimicrobial agents 47, 48-55. Wang, J., Wang, W., Kollman, P.A., and Case, D.A. (2006). Automatic atom type and bond type perception in molecular mechanical calculations. Journal of Molecular Graphics \& Modelling 25, 247-260. Warr, W.A. (2012). Scientific workflow systems: Pipeline Pilot and KNIME. Journal of Computer Aided Molecular Design 26, 801-804.

Williams, G.C., and Nesse, R.M. (1991). The dawn of Darwinian medicine. The Quarterly review of biology 66, 1-22.

Wróbel, A., Arciszewska, K., Maliszewski, D., and Drozdowska, D. (2020). Trimethoprim and other nonclassical antifolates an excellent template for searching modifications of dihydrofolate reductase enzyme inhibitors. The Journal of antibiotics 73, 5-27. 
Yeggoni, D.P., Gokara, M., Mark Manidhar, D., Rachamallu, A., Nakka, S., Reddy, C.S., and Subramanyam, R. (2014). Binding and Molecular Dynamics Studies of 7-Hydroxycoumarin Derivatives with Human Serum Albumin and Its Pharmacological Importance. Molecular Pharmaceutics 11, 1117-1131.

Zaritsky, A., Woldringh, C.L., Einav, M., and Alexeeva, S. (2006). Use of thymine limitation and thymine starvation to study bacterial physiology and cytology. Journal of bacteriology 188, 1667-1679.

Zhang, Y., Chen, Y., Zhang, D., Wang, L., Lu, T., and Jiao, Y. (2017). Discovery of Novel Potent VEGFR-2 Inhibitors Exerting Significant Antiproliferative Activity against Cancer Cell Lines. Journal of Medicinal Chemistry 61, 140-157.

Zhang, Y., Chen, Y., Zhang, D., Wang, L., Lu, T., and Jiao, Y. (2018). Discovery of Novel Potent VEGFR-2 Inhibitors Exerting Significant Antiproliferative Activity against Cancer Cell Lines. J Med Chem 61, 140157.

Zhang, Y., Jiao, Y., Xiong, X., Liu, H., Ran, T., Xu, J., Lu, S., Xu, A., Pan, J., Qiao, X., et al. (2015). Fragment virtual screening based on Bayesian categorization for discovering novel VEGFR-2 scaffolds. Molecular diversity 19, 895-913.

Zhang, Y., Yang, S., Jiao, Y., Liu, H., Yuan, H., Lu, S., Ran, T., Yao, S., Ke, Z., and Xu, J. An Integrated Virtual Screening Approach for VEGFR-2 Inhibitors. Journal of Chemical Information \& Modeling 53, 3163-3177. Zhang, Y., Yang, S., Jiao, Y., Liu, H., Yuan, H., Lu, S., Ran, T., Yao, S., Ke, Z., and Xu, J. (2013). An Integrated Virtual Screening Approach for VEGFR-2 Inhibitors. Journal of Chemical Information \& Modeling 53, 3163-3177. 


\section{Supplementary Figures:}
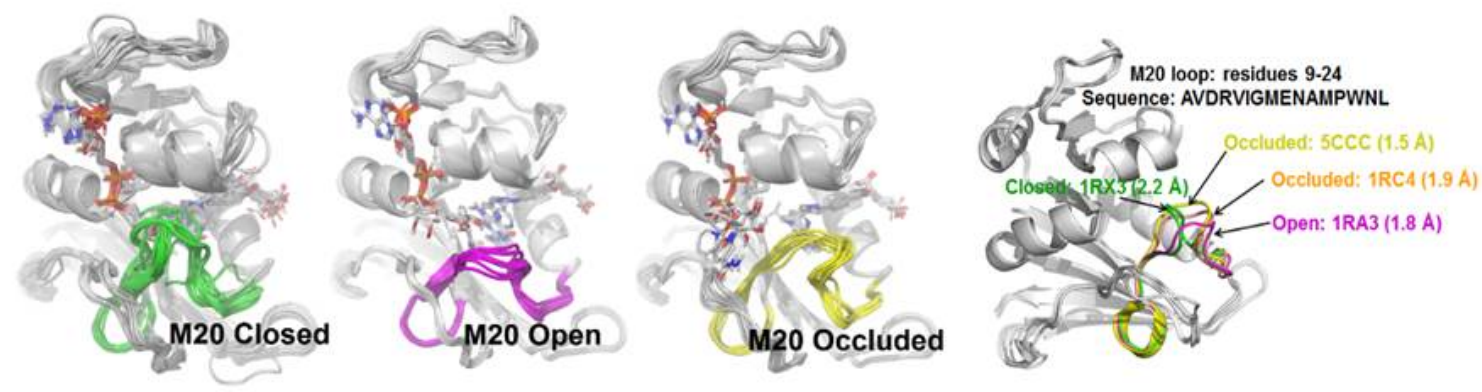

Figure S1 Three different types of M20 loops in E.coli DHFR. In the crystal structures of E.coli DHFR, the M20 loop (residues 9-24) has three major types of conformation (open, closed and occluded)(Falzone et al.) that are important for catalysis. 


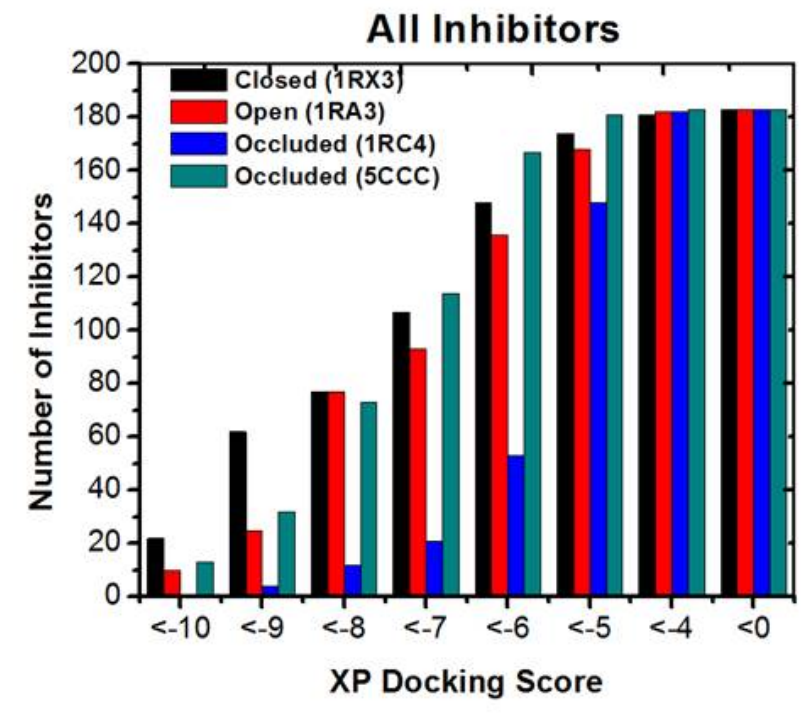

(A)

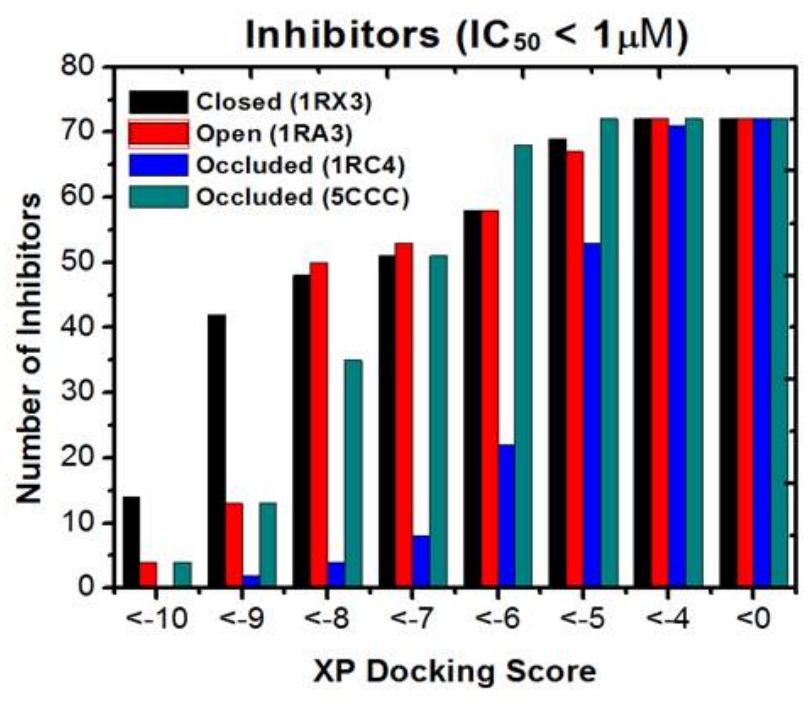

(B)

Figure S2. The number distribution of inhibitors with a given XP docking score. (A) All 183 inhibitors; (B) 72 Inhibitors with IC50 values less than $1 \mu M$. Lower scores correspond to stronger binding. 
bioRxiv preprint doi: https://doi.org/10.1101/2020.10.30.362582; this version posted May 25, 2021. The copyright holder for this preprint (which

was not certified by peer review) is the author/funder, who has granted bioRxiv a license to display the preprint in perpetuity. It is made available under aCC-BY-NC-ND 4.0 International license.

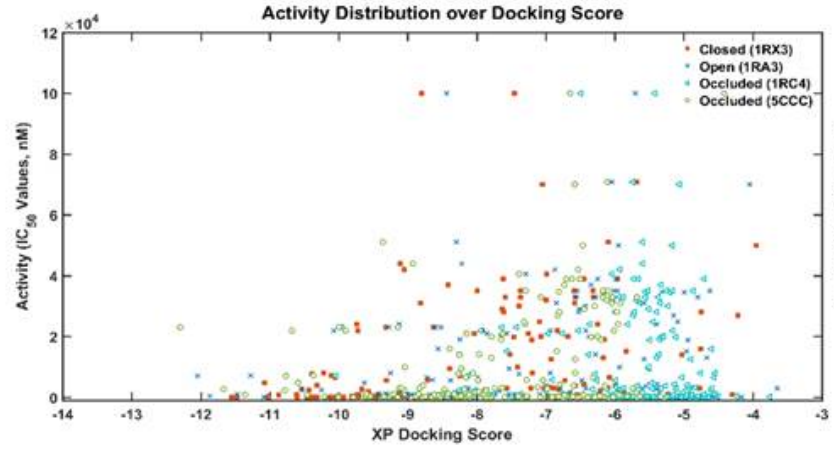

(A)

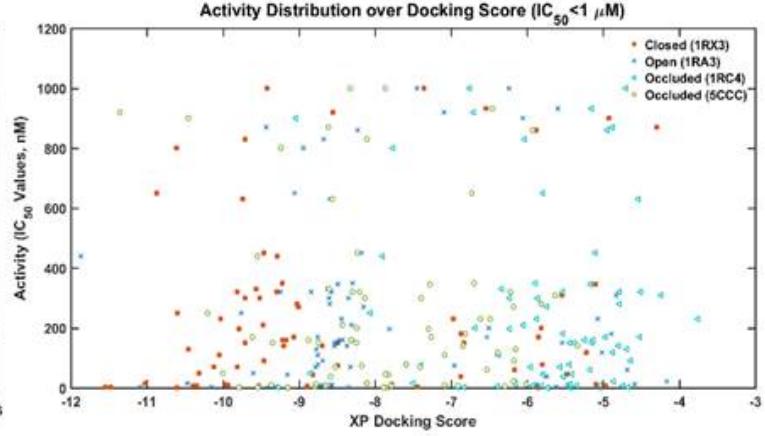

(B)

Figure S3. The scatter plot experimentally measured activities of DHFR inhibitors vs their XP docking score with various DHFR conformations as targets. (A) All 183 inhibitors; (B) 72 Inhibitors with $I C_{50}$ values less than $1 \mu M$. 


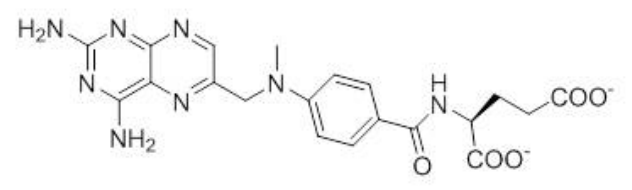

MTX

PDB: $1 \mathrm{RX} 3$

Kd: $0.021 \mathrm{nM}$

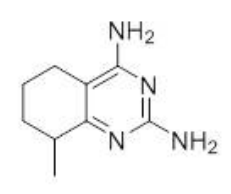

Cmpd 2

PDB: 3QYL

Kd: $230 \mathrm{nM}$

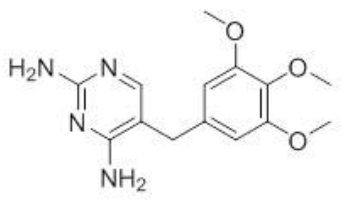

TMP

PDB: $1 \mathrm{RX} 3$

$\mathrm{Kd}: 1.2 \mathrm{nM}$<smiles>CC1CCc2c(N)nc(N)nc2C1</smiles>

Cmpd 4

PDB: $3 Q Y L$

Kd: $1000 \mathrm{nM}$

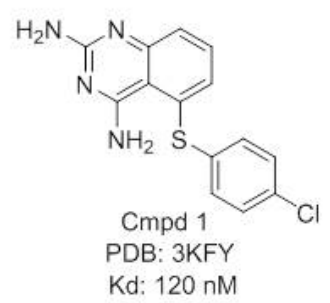<smiles>Nc1nc(N)c2c(n1)CCCC2</smiles><smiles>CC1CCCc2nc(N)nc(N)c21</smiles>

Cmpd 6

PDB: $3 Q Y L$

Kd: $43000 \mathrm{nM}$

Figure $\mathbf{S} 4$ Compounds used for building the binding affinity prediction model. 
bioRxiv preprint doi: https://doi.org/10.1101/2020.10.30.362582; this version posted May 25, 2021. The copyright holder for this preprint (which

was not certified by peer review) is the author/funder, who has granted bioRxiv a license to display the preprint in perpetuity. It is made available under aCC-BY-NC-ND 4.0 International license.

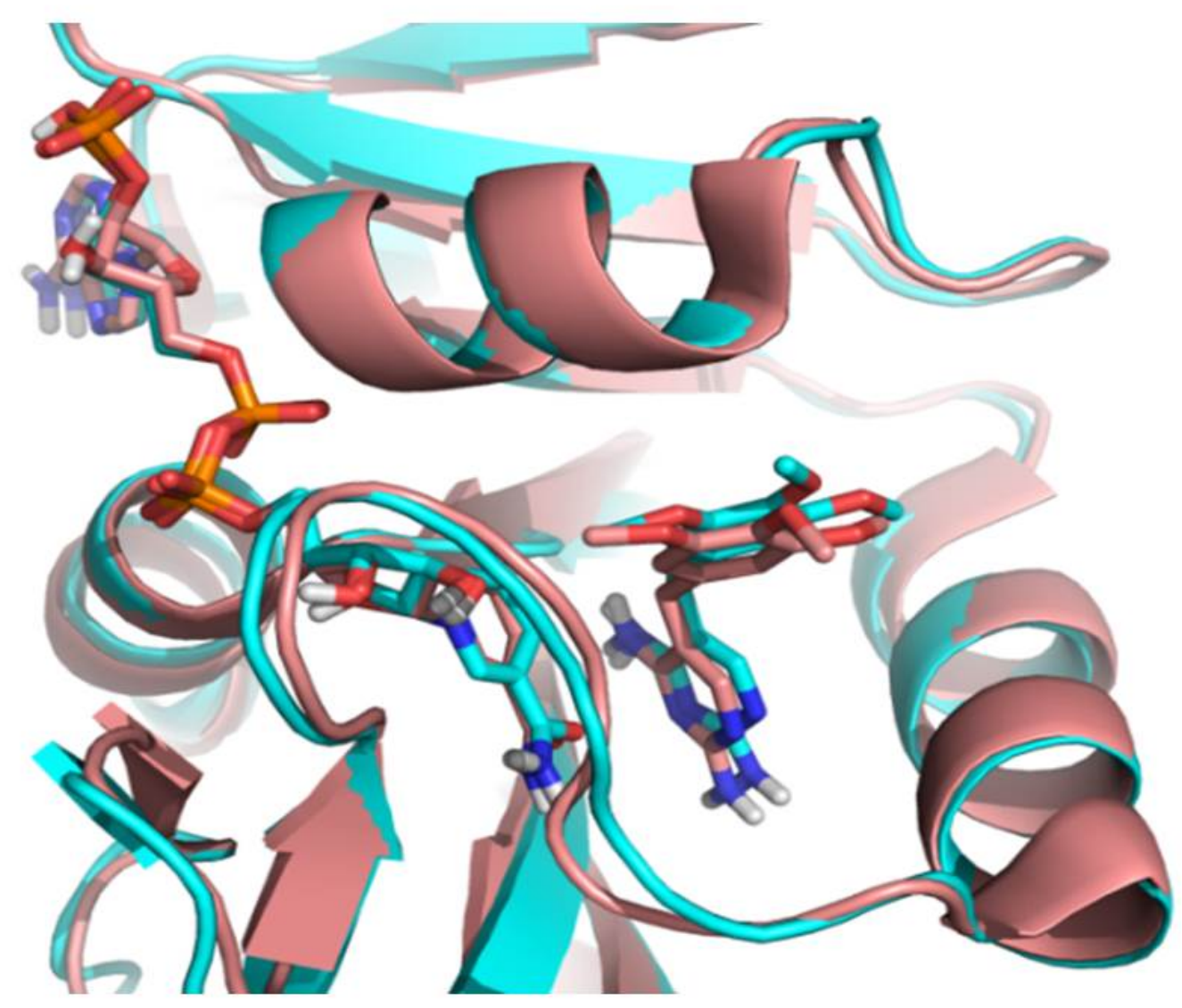

Figure S5 Comparison of docked TMP in E. coli DHFR (cyan, PDB 1RX3) with crystalized TMP with Staphylococcus aureus DHFR (salmon, PDB: 2W9G). 

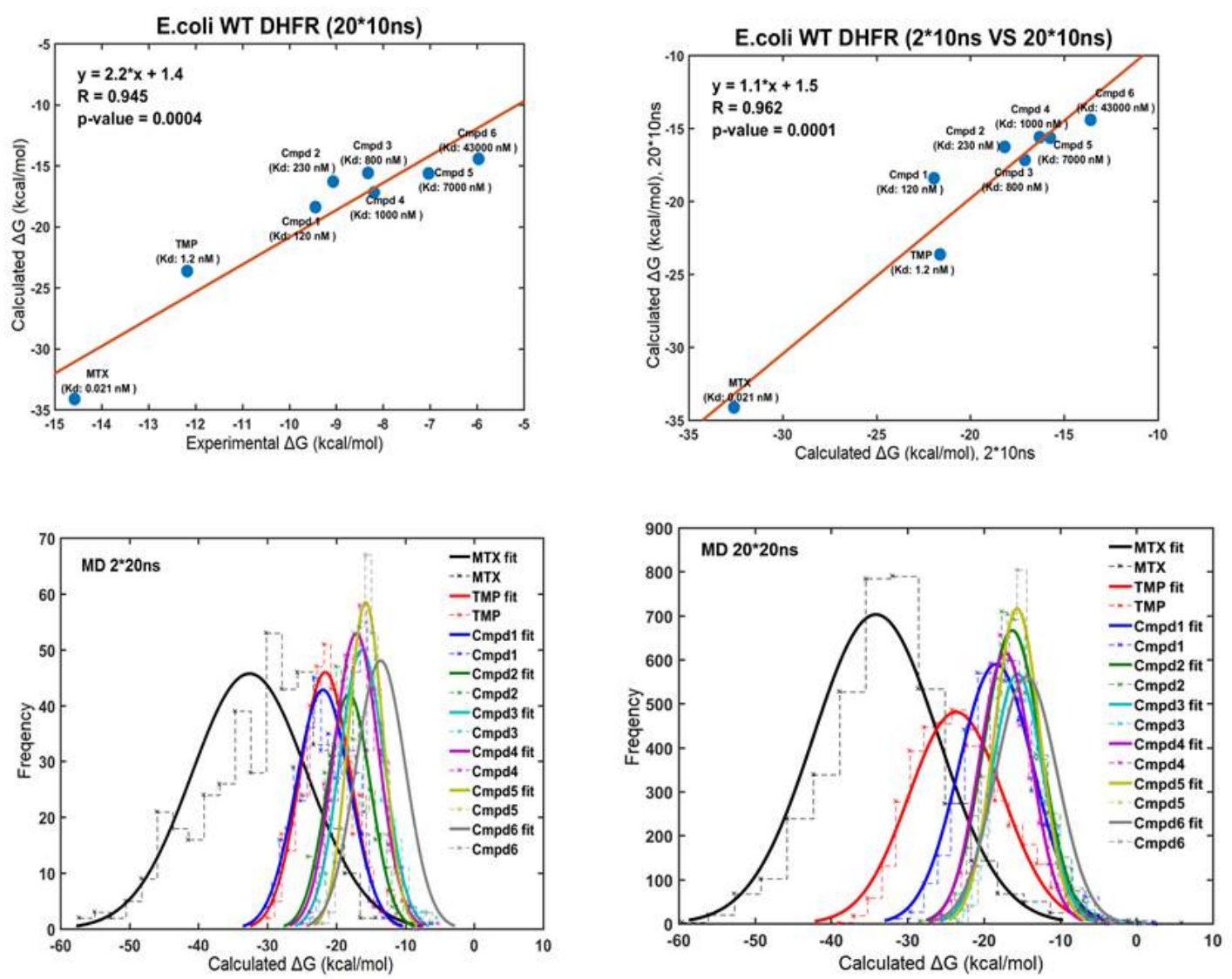

Figure S6 Linear correlation between the computational and experimental binding Gibbs free energies for eight compounds against WT E. coli DHFR (upper panel). Normal distribution of binding free energies (lower panel) 

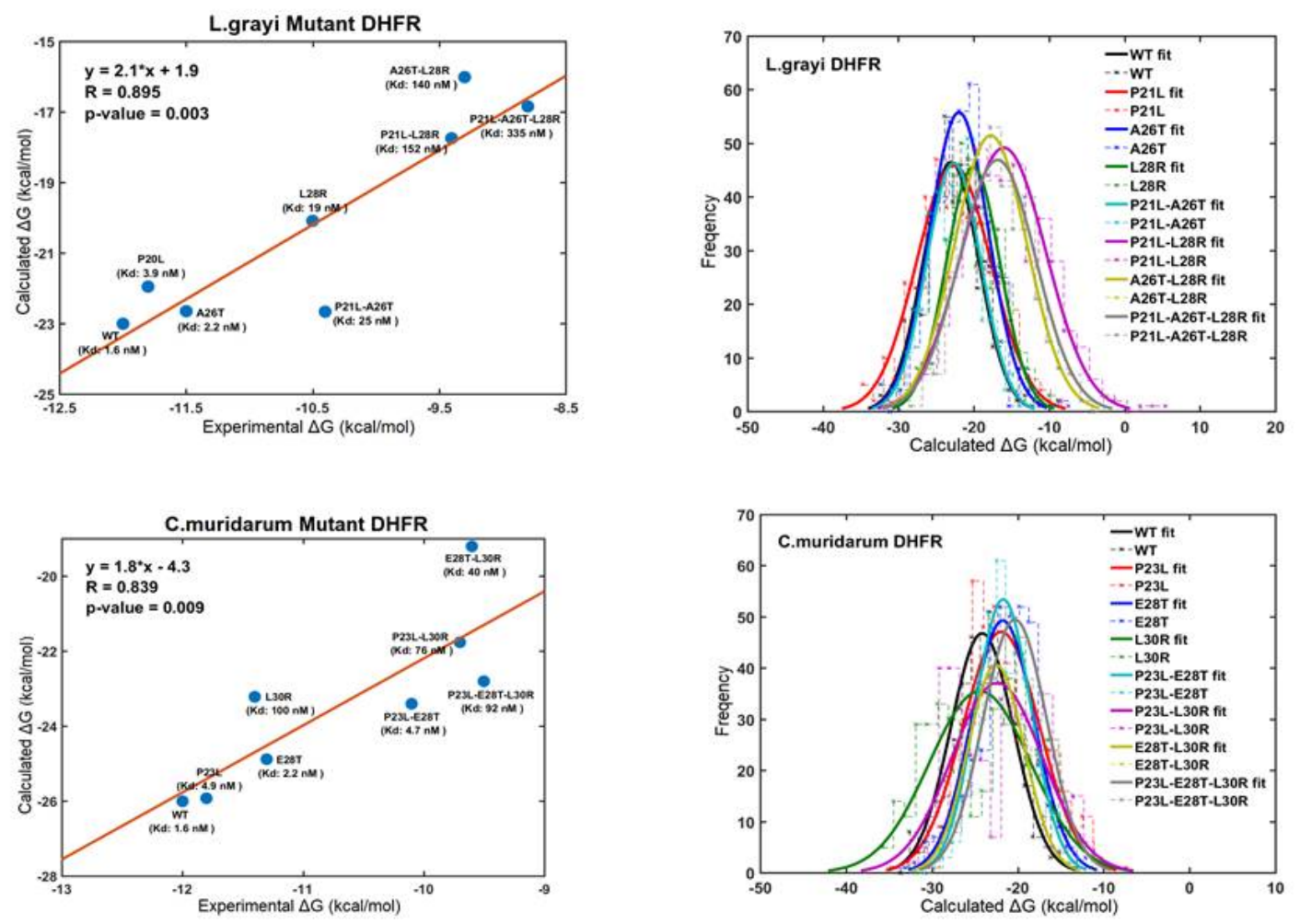

Figure $\mathbf{S 7}$ Correlation and linear models for the calculated and experimental binding Gibbs free energies for TMP against WT and mutant Listeria grayi (upper panel) and Chlamydia muridarum DHFR (lower panel). Normal distribution of binding free energies (right panel). 
bioRxiv preprint doi: https://doi.org/10.1101/2020.10.30.362582; this version posted May 25, 2021. The copyright holder for this preprint (which

was not certified by peer review) is the author/funder, who has granted bioRxiv a license to display the preprint in perpetuity. It is made available under aCC-BY-NC-ND 4.0 International license.
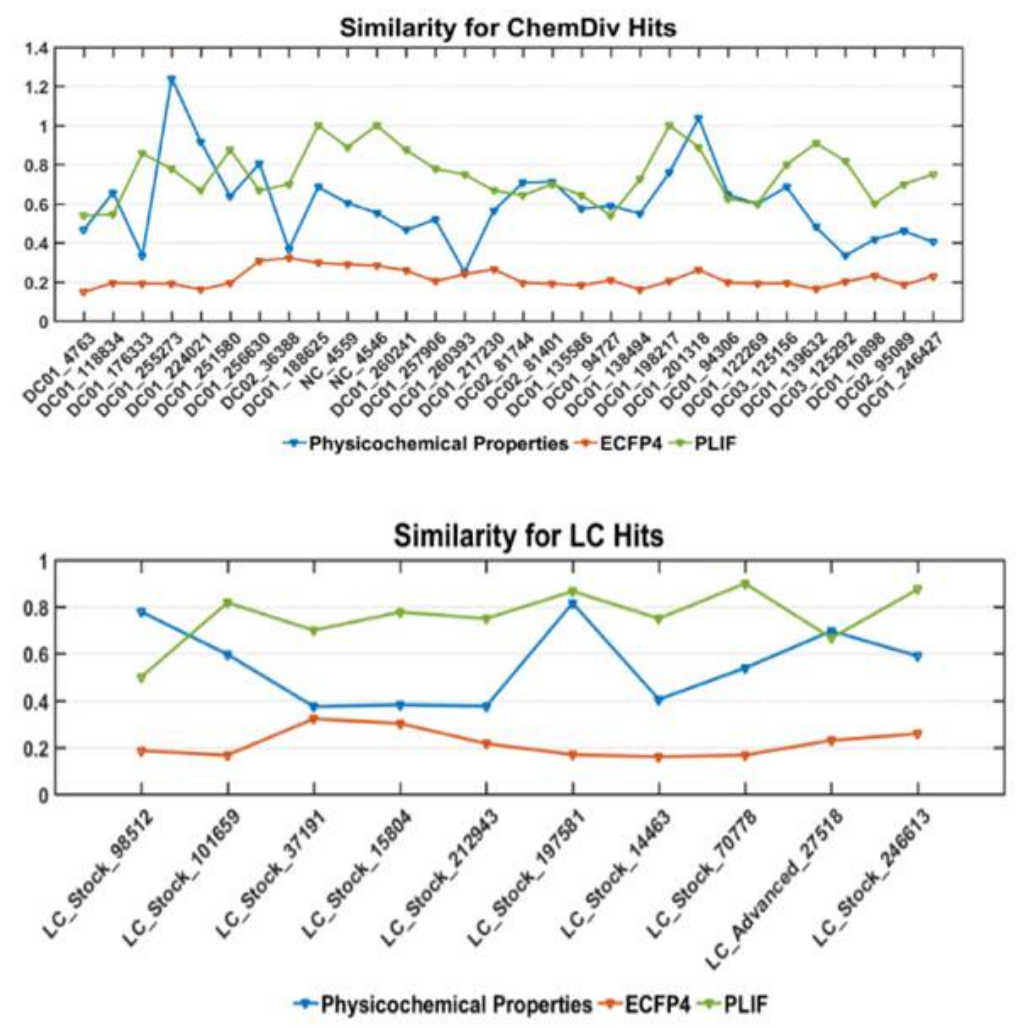

Figure S8 Similarity comparison between the selected hits with the known inhibitors using physicochemical properties, structure (represented by ECFP4) and PLIF. 
bioRxiv preprint doi: https://doi.org/10.1101/2020.10.30.362582; this version posted May 25, 2021. The copyright holder for this preprint (which

was not certified by peer review) is the author/funder, who has granted bioRxiv a license to display the preprint in perpetuity. It is made available under aCC-BY-NC-ND 4.0 International license.
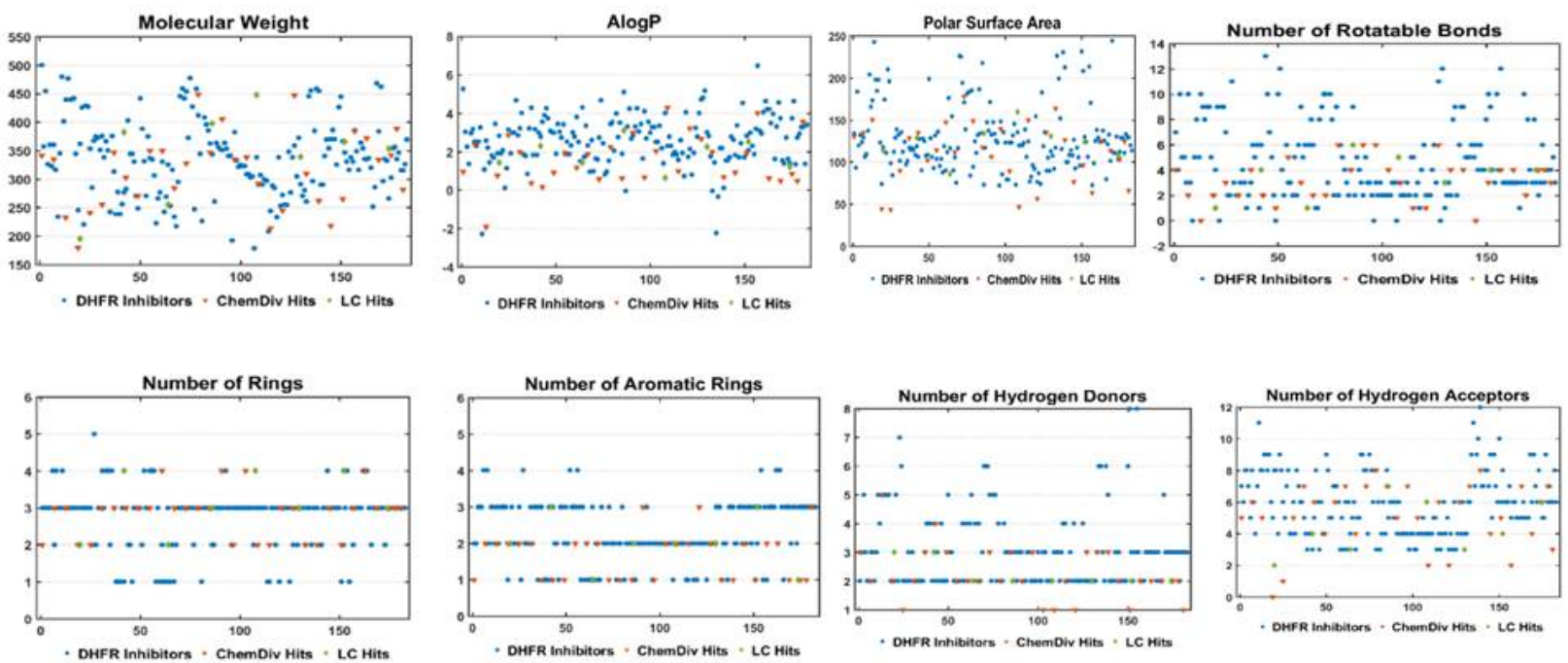

Figure S9 Two-dimensional chemical space of physiochemical properties for the selected hits with the known DHFR inhibitors. 

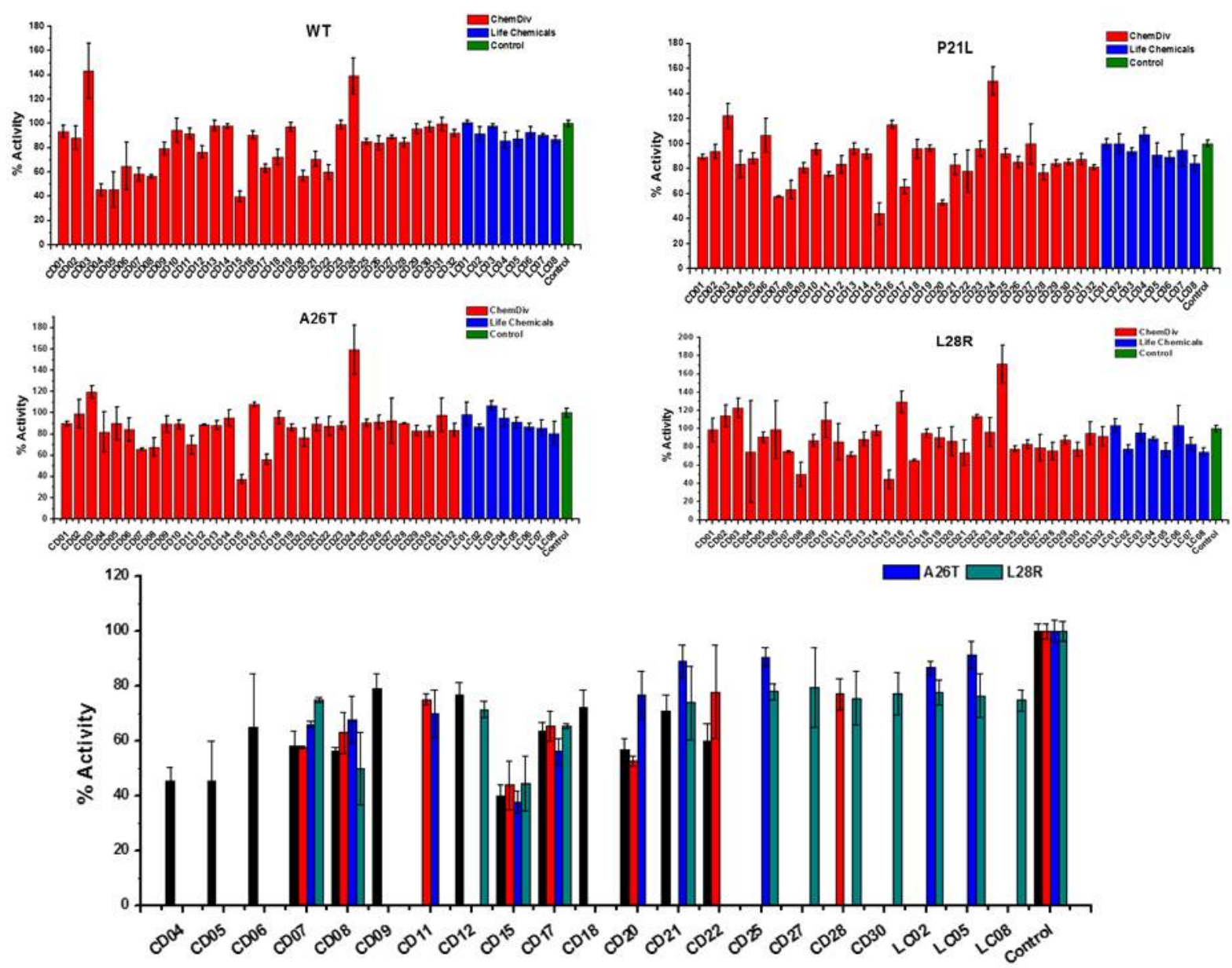

Figure S10 The initial inhibition rate of catalytic activity of the selected 40 hits against WT and three DHFR mutants at a single concentration of $200 \mu \mathrm{M}$. Each experiment was conducted in triplicates. 


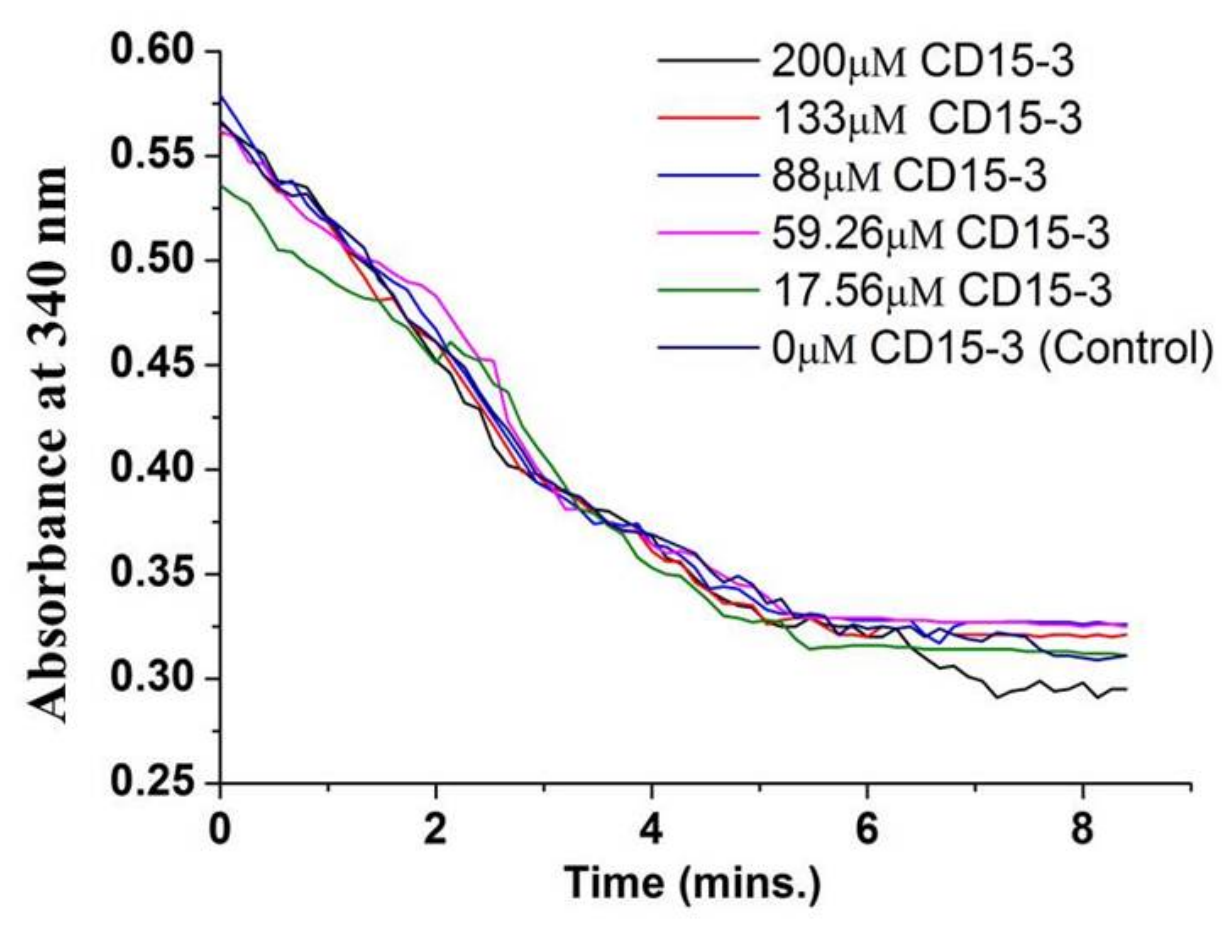

Figure S11: Activity assay for human DHFR in absence $(0 \mu M)$ and presence of CD15-3 (17.56 to $200 \mu \mathrm{M}$ ) carried out at $25^{\circ} \mathrm{C}$. Decrease in the fluorescence signal at $450 \mathrm{~nm}$ (which corresponds to conversion of NADPH and DHFR mediated formation of THF from DHF) was found to occur at all the concentrations of CD15-3 used. 


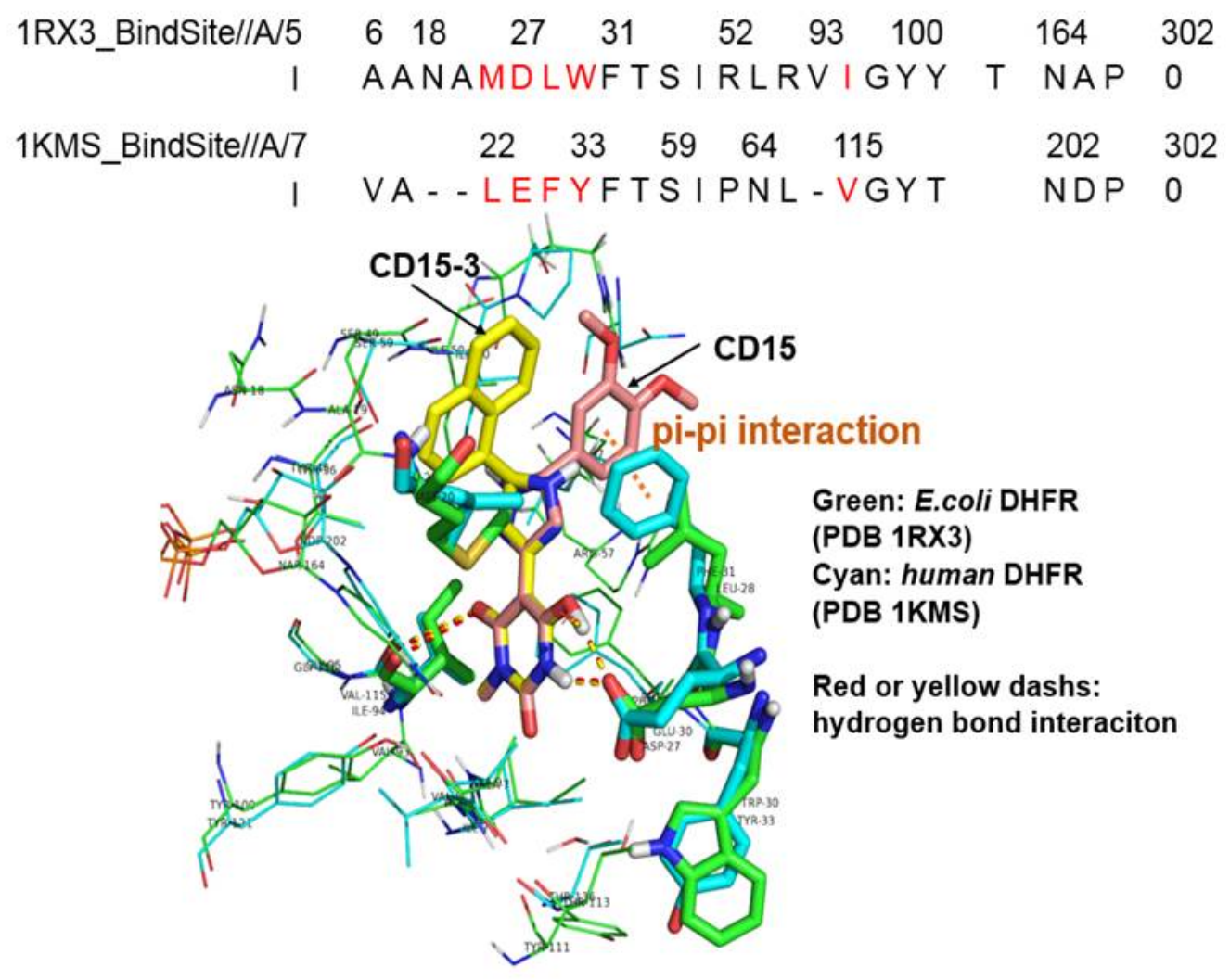

Figure S12: Sequence and structural alignment of the binding site for E.coli DHFR and human DHFR. The upper panel shows the binding site sequence alignment of E.coli DHFR (PDB 1RX3) and human DHFR (PDB IKMS), with amino acid/sequence differences shown in red colour. The lower panel demonstrates the structural comparison of binding site for E.coli DHFR (PDB 1RX3, colored green) and human DHFR (PDB IKMS, colored cyan). CD15-3(colored yellow) and CD15 (colored salmon) are docked to the binding site of human DHFR (PDB IKMS). The red dashes are the hydrogen bond for CD15-3 to E.coli DHFR and human DHFR. The yellow dashes are the hydrogen bond for the CD15 to E.coli DHFR and human DHFR. The orange dash shows the pi-pi interaction between CD15 and human DHFR (PDB IKMS). 


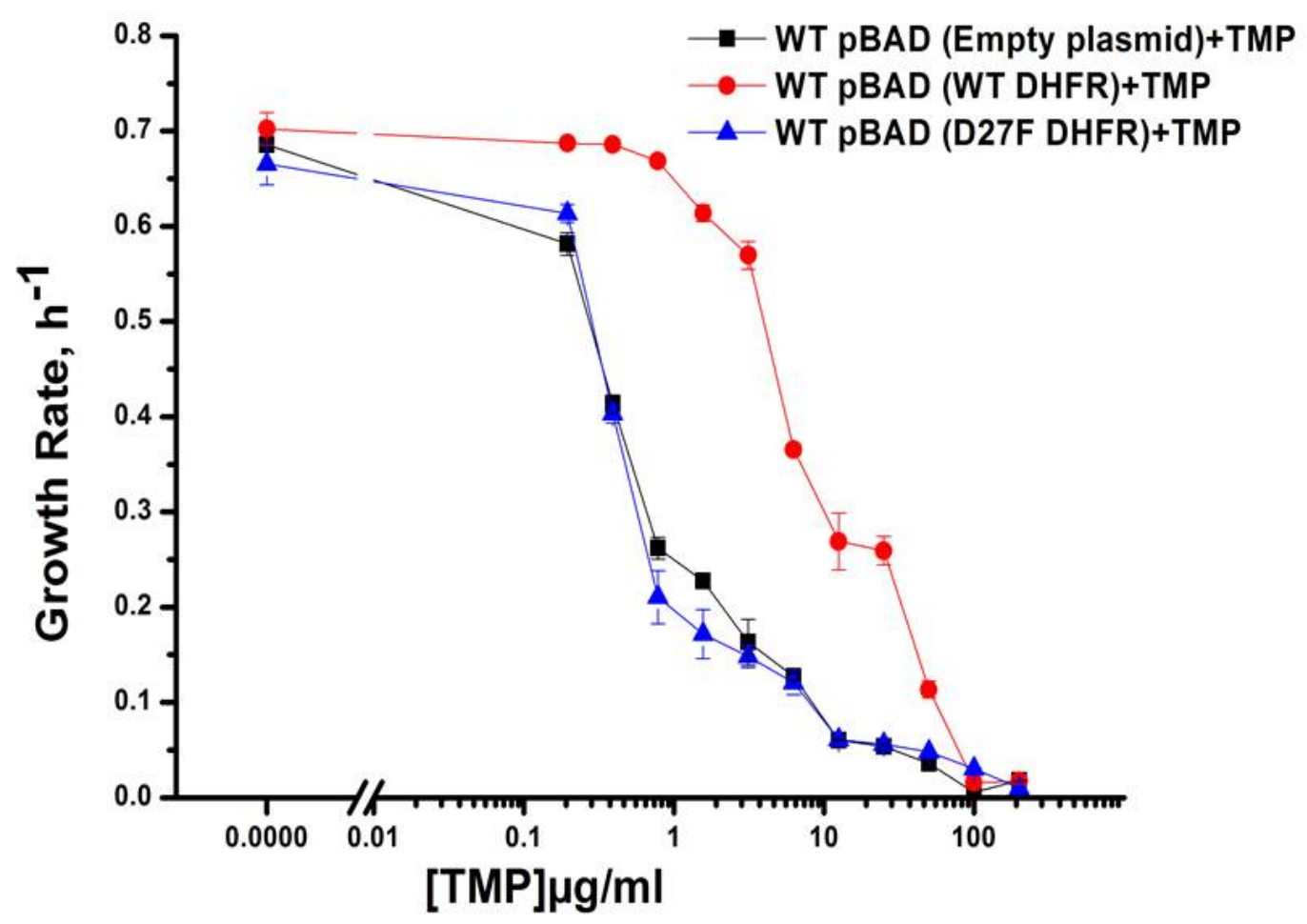

Figure S13: Growth rate profiles of WT E.coli cells with empty pBAD-plasmid and with WT DHFR and functionally inactive D27F mutant form of DHFR. Expression was induced using $0.005 \%$ Arabinose and cells were grown in M9 media. Plot shows overexpression of functional form of DHFR i.e. WT DHFR can recover the growth rates of cells growing in presence of TMP. Overexpression of $D 27 F$ failed to recover growth rates of TMP treated cells. 
bioRxiv preprint doi: https://doi.org/10.1101/2020.10.30.362582; this version posted May 25, 2021. The copyright holder for this preprint (which was not certified by peer review) is the author/funder, who has granted bioRxiv a license to display the preprint in perpetuity. It is made available under aCC-BY-NC-ND 4.0 International license. 